\title{
On a hybrid inclusion problem via hybrid boundary value conditions
}

\author{
S. Etemad', S. Pourrazi ${ }^{1,2}$ and Sh. Rezapour ${ }^{1,3^{*}}$
}

"Correspondence:
rezapourshahram@yahoo.ca;
sh.rezapour@azaruniv.ac.ir
${ }^{1}$ Department of Mathematics,
Azarbaijan Shahid Madani
University, Tabriz, Iran
${ }^{3}$ Department of Medical Research,
China Medical University Hospital,
China Medical University, Taichung,
Taiwan
Full list of author information is
available at the end of the article

available at the end of the article

\begin{abstract}
In this manuscript, the existence of solutions for a novel category of the fractional differential equation of hybrid type with hybrid boundary value conditions is studied. Also, we review the existence result for its related hybrid inclusion problem with hybrid conditions. In the end of the paper, two illustrative examples are given to demonstrate the consistency to our key results.
\end{abstract}

MSC: Primary 34A08; secondary 34A12

Keywords: Hybrid fractional differential equation; Inclusion problem; Fractional Caputo derivative; Dhage's fixed point

\section{Introduction}

As you know, we can introduce different kinds of modeling made by means of powerful and logical tools in mathematics. One of these tools is formulas and relations defined in the fractional calculus. Indeed, the importance and efficiency of this branch of mathematics has caused recent developments of FDEs or inclusions. Therefore, many researchers have studied some results on the properties of solutions for fractional BVPs under general boundary conditions. In this way, the authors use different approaches for their special goals. In other words, some recent published works show the importance of fractional differential equations in modeling of a variety of applied sciences (see, for example, [1-9]), numerical computations (see [10-15]), and different views on this field (see, for example, [16-51]).

Dhage and Lakshmikantham [52] designed a new category of differential equations called hybrid differential equations and studied the properties of solution for this type of differential equations. Two years later, Zhao et al. [53] provided an extension for Dhage's article to arbitrary real order and considered the related hybrid differential equation. Some years later, Hilal and Kajouni [54] studied the existence of some extremal solutions for the Caputo hybrid BVP given by

$$
\left\{\begin{array}{l}
{ }^{c} \mathcal{D}^{v}\left(\frac{\varrho(t)}{w(t, \varrho(t))}\right)=\Phi(t, \varrho(t))=0, \quad(t \in[0, T]), \\
a_{1}^{*} \frac{\varrho(0)}{w(0, \varrho(0))}+a_{2}^{*} \frac{\varrho(T)}{w(T, \varrho(T))}=a_{3}^{*},
\end{array}\right.
$$

(c) The Author(s) 2020. This article is licensed under a Creative Commons Attribution 4.0 International License, which permits use, sharing, adaptation, distribution and reproduction in any medium or format, as long as you give appropriate credit to the original author(s) and the source, provide a link to the Creative Commons licence, and indicate if changes were made. The images or other third party material in this article are included in the article's Creative Commons licence, unless indicated otherwise in a credit line to the material. If material is not included in the article's Creative Commons licence and your intended use is not permitted by statutory regulation or exceeds the permitted use, you will need to obtain permission directly from the copyright holder. To view a copy of this licence, visit http://creativecommons.org/licenses/by/4.0/. 
where $v \in(0,1), a_{1}^{*}, a_{2}^{*}$, and $a_{3}^{*}$ are three real constants with $a_{1}^{*}+a_{2}^{*} \neq 0$, and two functions $\Phi:[0, T] \times \mathbb{R} \rightarrow \mathbb{R}$ and $w:[0, T] \times \mathbb{R} \rightarrow \mathbb{R} \backslash\{0\}$ are continuous. After that, Baleanu et al. discussed existence theorems for the Caputo hybrid inclusion of arbitrary order

$$
\begin{aligned}
& { }^{c} \mathcal{D}^{v}\left(\frac{\varrho(t)}{w\left(t, \varrho(t), \mathcal{J}^{\alpha_{1}} \varrho(t), \ldots, \mathcal{J}^{\alpha_{n}} \varrho(t)\right)}\right) \in \Psi\left(t, \varrho(t), \mathcal{J}^{\beta_{1}} \varrho(t), \ldots, \mathcal{J}^{\beta_{k}} \varrho(t)\right), \\
& \quad(t \in[0,1])
\end{aligned}
$$

with boundary value conditions $\varrho(0)=\varrho_{0}^{*}$ and $\varrho(1)=\varrho_{1}^{*}, \mathcal{J}^{\gamma}$ is the Riemann-Liouville integral operator of fractional order $\gamma \in\left\{\alpha_{j}, \beta_{l}\right\} \subset(0, \infty)$ for $j=1, \ldots n$ and $l=1, \ldots, k$. Moreover, ${ }^{c} \mathcal{D}^{v}$ is the Caputo derivative operator of order $1<v \leq 2$ [55]. Recently, Derbazi et al. derived several uniqueness and existence theorems for the following BVP of hybrid type:

$$
{ }^{c} \mathcal{D}^{v}\left(\frac{\varrho(t)-g(t, \varrho(t))}{w(t, \varrho(t))}\right)=\Phi(t, \varrho(t)), \quad(t \in[0, T])
$$

with boundary conditions

$$
\left\{\begin{array}{l}
\left.a_{1}\left(\frac{\varrho(t)-g(t, \varrho(t))}{w(t, \varrho(t))}\right)\right|_{t=0}+\left.b_{1}\left(\frac{\varrho(t)-g(t, \varrho(t))}{w(t, \varrho(t))}\right)\right|_{t=T}=\lambda_{1}^{*}, \\
\left.a_{2}{ }^{c} \mathcal{D}^{\beta}\left(\frac{\varrho(t)-g(t, \varrho(t))}{w(t, \varrho(t))}\right)\right|_{t=\eta}+\left.b_{2}{ }^{c} \mathcal{D}^{\beta}\left(\frac{\varrho(t)-g(t, \varrho(t))}{w(t, \varrho(t))}\right)\right|_{t=T}=\lambda_{2}^{*},
\end{array}\right.
$$

where $v \in(1,2], \beta \in(0,1], \eta \in(0, T)$ and $a_{j}, b_{j}$, and $\lambda_{j}^{*}$ are real constants for $j=1,2$ [56]. Also, all fractional derivatives given in the above problem are of Caputo type. The authors proved their main results based on Dhage's fixed point result for three operators [56].

By mixing and generalizing the above ideas, we are going to derive a new existence theorem for solution functions $\varrho(t)$ of the following hybrid differential equation of order $v$ :

$$
{ }^{c} \mathcal{D}^{v}\left(\frac{\varrho(t)}{w(t, \varrho(t))}\right)=\Phi(t, \varrho(t)), \quad(t \in[0,1])
$$

with hybrid boundary conditions

$$
\left\{\begin{array}{l}
\left.\left(\frac{\varrho(t)}{w(t, \varrho(t))}\right)\right|_{t=0}=0 \\
\left.\mathcal{D}\left(\frac{\varrho(t)}{w(t, \varrho(t))}\right)\right|_{t=0}=-\left.\left(\frac{\varrho(t)}{w(t, \varrho(t))}\right)\right|_{t=1}-\left.\mathcal{D}\left(\frac{\varrho(t)}{w(t, \varrho(t))}\right)\right|_{t=1} \\
\left.\mathcal{D}^{2}\left(\frac{\varrho(t)}{w(t, \varrho(t))}\right)\right|_{t=0}=-\left.\mathcal{D}^{2}\left(\frac{\varrho(t)}{w(t, \varrho(t))}\right)\right|_{t=1}-\left.^{c} \mathcal{D}^{\theta}\left(\frac{\varrho(t)}{w(t, \varrho(t))}\right)\right|_{t=1}
\end{array}\right.
$$

where $v \in(2,3], \theta \in(1,2], \mathcal{D}=\frac{\mathrm{d}}{\mathrm{d} t}, \mathcal{D}^{2}=\frac{\mathrm{d}^{2}}{\mathrm{~d} t^{2}}$, and ${ }^{c} \mathcal{D}^{\beta}$ is the Caputo derivative of order $\beta \in\{v, \theta\}$. Moreover, $\Phi$ is a real-valued continuous function defined on $[0,1] \times \mathbb{R}$ and $w \in C_{\mathbb{R}}([0,1] \times \mathbb{R})$ is a nonzero function. In the following, we intend to study the related hybrid inclusion problem

$$
{ }^{c} \mathcal{D}^{v}\left(\frac{\varrho(t)}{w(t, \varrho(t))}\right) \in \Psi(t, \varrho(t)), \quad(t \in[0,1])
$$


with hybrid boundary value conditions

$$
\left\{\begin{array}{l}
\left.\left(\frac{\varrho(t)}{w(t, \varrho(t))}\right)\right|_{t=0}=0 \\
\left.\mathcal{D}\left(\frac{\varrho(t)}{w(t, \varrho(t))}\right)\right|_{t=0}=-\left.\left(\frac{\varrho(t)}{w(t, \varrho(t))}\right)\right|_{t=1}-\left.\mathcal{D}\left(\frac{\varrho(t)}{w(t, \varrho(t))}\right)\right|_{t=1}, \\
\left.\mathcal{D}^{2}\left(\frac{\varrho(t)}{w(t, \varrho(t))}\right)\right|_{t=0}=-\left.\mathcal{D}^{2}\left(\frac{\varrho(t)}{w(t, \varrho(t))}\right)\right|_{t=1}-\left.{ }^{c} \mathcal{D}^{\theta}\left(\frac{\varrho(t)}{w(t, \varrho(t))}\right)\right|_{t=1}
\end{array}\right.
$$

where $\Psi:[0,1] \times \mathbb{R} \rightarrow \mathcal{P}(\mathbb{R})$ is a given set-valued map via some properties.

To reach the main purposes of this manuscript, the techniques of the fixed point theory are employed to prove the theoretical results. Our investigations are two fold: we first deal with a hybrid differential equation and then with its related hybrid differential inclusion. It is worth mentioning that the proposed hybrid problems (1)-(2) and (3)-(4) differ from the newly defined ones. In both hybrid problems (1)-(2) and (3)-(4), we consider three boundary conditions of hybrid type in terminal points. We believe that our hybrid problems involve some particular cases, which can be extended to more general hybrid problems. The fractional hybrid modeling is of great significance in different engineering fields, and it can be a unique idea for the future combined research between various applied sciences (see [57]). As a practical example of applicability of our results, we can point out to our newly published work [58]. The fractional hybrid problem given in [58] is a particular case of the proposed hybrid problem (1)-(2) in this work, in which a fractional hybrid modeling of a thermostat is simulated.

The content of this article is arranged as follows: In Sect. 2, some required concepts in this regard are recalled. Section 3 is devoted to proving the main theorems relying on some mathematical inequalities and two versions of fixed point theorems due to Dhage. At the end of the paper, we give two illustrative examples to support the applicability of our findings.

\section{Preliminaries}

Prior to proceeding to the main objectives, we here recall some essential auxiliary concepts which are needed in the sequel. Let $v \in \mathbb{R}^{+}$so that $v \in(n-1, n]$ and assume that the realvalued function $\varrho$ is integrable on $[a, b]$. In this case, the Riemann-Liouville integral of the function $\varrho$ of order $v$ is given by

$$
\mathcal{J}^{v} \varrho(t)=\int_{0}^{t} \frac{(t-\tau)^{\nu-1}}{\Gamma(v)} \varrho(\tau) \mathrm{d} \tau
$$

provided that the integral is finite-valued $[59,60]$. With the same assumptions, the Caputo derivative of order $v$ for a function $\varrho \in C_{\mathbb{R}}^{(n)}([a, b])$ is given by

$$
{ }^{c} \mathcal{D}^{v} \varrho(t)=\int_{0}^{t} \frac{(t-\tau)^{n-v-1}}{\Gamma(n-v)} \varrho^{(n)}(\tau) \mathrm{d} \tau,
$$

provided that the integral is finite-valued and $n=1+[v][59,60]$. According to the existing propositions, if we solve the fractional homogeneous differential equation ${ }^{c} \mathcal{D}_{0}^{v} \varrho(t)=0$, then its general solution is obtained as $\varrho(t)=b_{0}+b_{1} t+b_{2} t^{2}+\cdots+b_{n-1} t^{n-1}$, where $b_{0}, \ldots, b_{n-1} \in \mathbb{R}$ and $n=1+[v][59,60]$. For every $\varrho \in C_{\mathbb{R}}([0, T])$ with $T>0$, the linear 
equation

$$
\left(\mathcal{J}^{\nu c} \mathcal{D}^{\nu}\right) \varrho(t)=\varrho(t)+\sum_{j=0}^{n-1} b_{j} t^{j}=\varrho(t)+b_{0}+b_{1} t+b_{2} t^{2}+\cdots+b_{n-1} t^{n-1}
$$

holds, where $b_{0}, \ldots, b_{n-1} \in \mathbb{R}$ and $n=1+[v][59,60]$.

Here, consider the normed space $\left(\mathcal{X},\|\cdot\|_{\mathcal{X}}\right)$. Then the collection of all subsets of $\mathcal{X}$, the collection of closed subsets of $\mathcal{X}$, the collection of bounded subsets of $\mathcal{X}$, the collection of convex subsets of $\mathcal{X}$, and the collection of compact subsets of $\mathcal{X}$ are denoted by $\mathcal{P}(\mathcal{X})$, $\mathcal{P}_{c l}(\mathcal{X}), \mathcal{P}_{b}(\mathcal{X}), \mathcal{P}_{c v}(\mathcal{X})$, and $\mathcal{P}_{c p}(\mathcal{X})$, respectively. A set-valued map $\Psi$ is convex-valued if for each $\varrho \in \mathcal{X}$ the set $\Psi(\varrho)$ is convex. The set-valued map $\Psi$ has an upper semi-continuity property whenever, for every $\varrho^{*} \in \mathcal{X}, \Psi\left(\rho^{*}\right)$ belongs to $\mathcal{P}_{c l}(\mathcal{X})$ and for each open set $\mathcal{O}$ with $\Psi\left(\varrho^{*}\right) \subset \mathcal{O}$ there is at least a neighborhood $\mathcal{V}_{0}^{*}$ of $\varrho^{*}$ provided that $\Psi\left(\mathcal{V}_{0}^{*}\right) \subseteq \mathcal{O}$ [61]. Moreover, $\varrho^{*} \in \mathcal{X}$ is a fixed point for the set-valued map $\Psi: \mathcal{X} \rightarrow \mathcal{P}(\mathcal{X})$ whenever $\varrho^{*} \in$ $\Psi\left(\varrho^{*}\right)$ [61]. The notation $\operatorname{Fix}(\Psi)$ denotes the set of all fixed points of set-valued $\Psi$ [61].

Consider the metric space $\mathcal{X}$ furnished with the metric $d_{\mathcal{X}}$. For every $E_{1}, E_{2} \in \mathcal{P}(\mathcal{X})$, the Pompeiu-Hausdorff metric $\mathrm{PH}_{d}: \mathcal{P}(\mathcal{X}) \times \mathcal{P}(\mathcal{X}) \rightarrow \mathbb{R} \cup\{\infty\}$ is defined by

$$
\mathrm{PH}_{d_{\mathcal{X}}}\left(E_{1}, E_{2}\right)=\max \left\{\sup _{a_{1} \in E_{1}} d_{\mathcal{X}}\left(a_{1}, E_{2}\right), \sup _{a_{2} \in E_{2}} d_{\mathcal{X}}\left(E_{1}, a_{2}\right)\right\}
$$

where $d_{\mathcal{X}}\left(E_{1}, a_{2}\right)=\inf _{a_{1} \in E_{1}} d_{\mathcal{X}}\left(a_{1}, a_{2}\right)$ and $d_{\mathcal{X}}\left(a_{1}, E_{2}\right)=\inf _{a_{2} \in E_{2}} d_{\mathcal{X}}\left(a_{1}, a_{2}\right)$ [61]. We say that the set-valued function $\Psi: \mathcal{X} \rightarrow \mathcal{P}_{c l}(\mathcal{X})$ is Lipschitzian if $\operatorname{PH}_{d_{\mathcal{X}}}\left(\Psi\left(\varrho_{1}\right), \Psi\left(\varrho_{2}\right)\right) \leq$ $l^{*} d_{\mathcal{X}}\left(\varrho_{1}, \varrho_{2}\right)$ holds for each $\varrho_{1}, \varrho_{2} \in \mathcal{X}$, where $l^{*}>0$ is a Lipschitz constant. A Lipschitz map $\Psi$ is said to be a contraction whenever $0<l^{*}<1[61]$. Furthermore, $\Psi:[0,1] \rightarrow \mathcal{P}_{c l}(\mathbb{R})$ is a measurable function if the mapping $t \longmapsto d_{\mathcal{X}}(r, \Psi(t))=\inf \{|r-\lambda|: \lambda \in \Psi(t)\}$ is measurable for all $r \in \mathbb{R}[61,62]$. The graph of $\Psi: \mathcal{X} \rightarrow \mathcal{P}_{c l}(\mathcal{Q})$ is defined by $\operatorname{Graph}(\Psi)=\left\{\left(\varrho_{1}, \varrho_{2}\right) \in\right.$ $\left.\mathcal{X} \times \mathcal{Q}: s^{*} \in \Psi(\varrho)\right\}[61]$. Note that the graph of $\Psi$ is closed if for arbitrary sequences $\left\{\varrho_{n}\right\}_{n \geq 1}$ belonging to $\mathcal{X}$ and $\left\{s_{n}\right\}_{n \geq 1}$ belonging to $\mathcal{Q}$ with $\varrho_{n} \rightarrow z_{0}, s_{n} \rightarrow s_{0}$, and $s_{n} \in \Psi\left(\varrho_{n}\right)$, we have $s_{0} \in \Psi\left(\varrho_{0}\right)[62]$.

A set-valued operator $\Psi$ has the complete continuity property if the set $\Psi(\mathcal{W})$ has the relative compactness property for all $\mathcal{W} \in \mathcal{P}_{b}(\mathcal{X})$. Let $\Psi: \mathcal{X} \rightarrow \mathcal{P}_{c l}(\mathcal{Q})$ have the upper semi-continuity property. Then $\operatorname{Graph}(\Psi) \subseteq \mathcal{X} \times \mathcal{Q}$ is a closed set. On the other hand, assume that $\Psi$ has a closed graph with the complete continuity property. Then $\Psi$ has the upper semi-continuity property [61]. We say that $\Psi:[0,1] \times \mathbb{R} \rightarrow \mathcal{P}(\mathbb{R})$ is a Caratheodory set-valued map if the mapping $\varrho \mapsto \Psi(t, \varrho)$ is upper semi-continuous for almost all $t \in$ $[0,1]$ and $t \mapsto \Psi(t, \varrho)$ is measurable for each $\varrho \in \mathbb{R}[61,62]$. In addition, a Caratheodory set-valued map $\Psi:[0,1] \times \mathbb{R} \rightarrow \mathcal{P}(\mathbb{R})$ is called $\mathcal{L}^{1}$-Caratheodory if for each $r>0$ there is $\phi_{r} \in \mathcal{L}_{\mathbb{R}^{+}}^{1}([0,1])$ provided that

$$
\|\Psi(t, \varrho)\|=\sup _{t \in[0,1]}\{|q|: q \in \Psi(t, \varrho)\} \leq \phi_{r}(t)
$$

for almost all $t \in[0,1]$ and for each $|\varrho| \leq r[61,62]$. The collection of all selections of $\Psi$ at $\varrho \in C_{\mathbb{R}}([0,1])$ is defined by

$$
(\mathcal{S E L})_{\Psi, \varrho}:=\left\{\vartheta \in \mathcal{L}_{\mathbb{R}}^{1}([0,1]): \vartheta(t) \in \Psi(t, \varrho(t)), \quad \text { a.e. } t \in[0,1]\right\}
$$


$[61,62]$. As it has been proved before in [61], $(\mathcal{S E L})_{\Psi, \varrho} \neq \emptyset$ for all $\varrho \in C_{\mathcal{X}}([0,1])$ whenever $\operatorname{dim} \mathcal{X}<\infty$. We need the following results.

Theorem 1 ([63]) Let $\mathcal{X}$ be a Banach algebra, $\mathcal{V}_{\varepsilon}(0)$ and $\overline{\mathcal{V}}_{\varepsilon}(0)$ be an open ball and its closure for all $\varepsilon \in \mathbb{R}^{+}$, respectively. Assume that $\Upsilon_{1}: \mathcal{X} \rightarrow \mathcal{X}$ and $\Upsilon_{2}: \overline{\mathcal{V}}_{\varepsilon}(0) \rightarrow \mathcal{X}$ are two operators satisfying:

(a1) $\Upsilon_{1}$ is Lipschitzian where $l^{*}$ is a Lipschitz constant,

(a2) $\Upsilon_{2}$ is completely continuous,

(a3) $l^{*} \mathcal{K}_{0}^{*}<1$, where $\mathcal{K}_{0}^{*}=\left\|\Upsilon_{2}\left(\overline{\mathcal{V}}_{\varepsilon}(0)\right)\right\|_{\mathcal{X}}=\sup \left\{\left\|\Upsilon_{2} \varrho\right\|_{\mathcal{X}}: \varrho \in \overline{\mathcal{V}}_{\varepsilon}(0)\right\}$.

Then either (b1) there exists $v^{*} \in \mathcal{X}$ with $\left\|v^{*}\right\|_{\mathcal{X}}=\varepsilon$ so that $\mu \Upsilon_{1} v^{*} \Upsilon_{2} v^{*}=v^{*}$ for some $0<$ $\mu<1$ or (b2) the equation $\Upsilon_{1} \varrho \Upsilon_{2} \varrho=\varrho$ has a solution belonging to $\overline{\mathcal{V}}_{\varepsilon}(0)$.

Theorem 2 ([64]) Assume that $\mathcal{X}$ is a separable Banach space, $\Psi:[0,1] \times \mathcal{X} \rightarrow \mathcal{P}_{c p, c v}(\mathcal{X})$ is an $\mathcal{L}^{1}$-Carathéodory multifunction and $\Xi: \mathcal{L}_{\mathcal{X}}^{1}([0,1]) \rightarrow C_{\mathcal{X}}([0,1])$ is a linear continuous mapping. Then $\Xi \circ(\mathcal{S E L})_{\Psi}: C_{\mathcal{X}}([0,1]) \rightarrow \mathcal{P}_{c p, c v}\left(C_{\mathcal{X}}([0,1])\right)$ is an operator which belongs to $C_{\mathcal{X}}([0,1]) \times C_{\mathcal{X}}([0,1])$ defined by $\varrho \mapsto\left(\Xi \circ(\mathcal{S E L})_{\Psi}\right)(\varrho)=\Xi\left((\mathcal{S E L})_{\Psi, \varrho}\right)$ having the closed graph.

Theorem 3 ([65]) Let $\mathcal{X}$ be a Banach algebra. Assume that there are a set-valued map $\Upsilon_{2}: \mathcal{X} \rightarrow \mathcal{P}_{c p, c v}(\mathcal{X})$ and a single-valued map $\Upsilon_{1}: \mathcal{X} \rightarrow \mathcal{X}$ satisfying:

(a'1) $\Upsilon_{1}$ is Lipschitzian where $l^{*}$ is a Lipschitz constant,

(a'2) $\Upsilon_{2}$ is compact and upper semi-continuous,

$\left(a^{\prime} 3\right) 2 l^{*} \mathcal{K}_{0}^{*}<1$ with $\mathcal{K}_{0}^{*}=\left\|\Upsilon_{2}(\mathcal{X})\right\|$.

Then either $\left(b^{\prime} 1\right) \mathcal{Y}^{*}=\left\{v^{*} \in \mathcal{X} \mid \mu v^{*} \in \Upsilon_{1} v^{*} \Upsilon_{2} v^{*}, \mu>1\right\}$ is an unbounded set or ( $\left.b^{\prime} 2\right)$ there exists a solution belonging to $\mathcal{X}$ for the inclusion $\varrho \in \Upsilon_{1} \varrho \Upsilon_{2} \varrho$.

\section{Main results}

In this part of the paper, we intend to state our main theoretical findings on the existence results. To reach this aim, we consider $\mathcal{X}=\left\{\varrho(t): \varrho(t) \in C_{\mathbb{R}}([0,1])\right\}$ equipped with the supremum norm $\|\varrho\|_{\mathcal{X}}=\sup _{t \in[0,1]}|\varrho(t)|$ and the multiplication action on the space $\mathcal{X}$ defined by $\left(\varrho \cdot \varrho^{\prime}\right)(t)=\varrho(t) \varrho^{\prime}(t)$ for all $\varrho, \varrho^{\prime} \in \mathcal{X}$. Then an ordered triple $\left(\mathcal{X},\|\cdot\|_{\mathcal{X}}, \cdot\right)$ is a Banach algebra. In this moment, we present an essential lemma which converts fractional BVP (1)-(2) into integral equation.

Lemma 4 Assume that $g$ belongs to $\mathcal{X}$. Then $\varrho_{0}$ is a solution function for the hybrid equation of fractional order

$$
{ }^{c} \mathcal{D}^{v}\left(\frac{\varrho(t)}{w(t, \varrho(t))}\right)=g(t) \quad(v \in(2,3], \theta \in(1,2], t \in[0,1])
$$

with hybrid boundary value conditions

$$
\begin{aligned}
& \left.\left(\frac{\varrho(t)}{w(t, \varrho(t))}\right)\right|_{t=0}=0, \\
& \left.\mathcal{D}\left(\frac{\varrho(t)}{w(t, \varrho(t))}\right)\right|_{t=0}=-\left.\left(\frac{\varrho(t)}{w(t, \varrho(t))}\right)\right|_{t=1}-\left.\mathcal{D}\left(\frac{\varrho(t)}{w(t, \varrho(t))}\right)\right|_{t=1} \\
& \left.\mathcal{D}^{2}\left(\frac{\varrho(t)}{w(t, \varrho(t))}\right)\right|_{t=0}=-\left.\mathcal{D}^{2}\left(\frac{\varrho(t)}{w(t, \varrho(t))}\right)\right|_{t=1}-\left.{ }^{c} \mathcal{D}^{\theta}\left(\frac{\varrho(t)}{w(t, \varrho(t))}\right)\right|_{t=1}
\end{aligned}
$$


iff the function $\varrho_{0}$ is a solution for the following integral equation of fractional order:

$$
\begin{aligned}
\varrho(t)= & w(t, \varrho(t))\left[\int_{0}^{t} \frac{(t-\tau)^{\nu-1}}{\Gamma(\nu)} g(\tau) \mathrm{d} \tau-\frac{t}{3} \int_{0}^{1} \frac{(1-\tau)^{\nu-1}}{\Gamma(v)} g(\tau) \mathrm{d} \tau\right. \\
& -\frac{t}{3} \int_{0}^{1} \frac{(1-\tau)^{\nu-2}}{\Gamma(v-1)} g(\tau) \mathrm{d} \tau+\frac{\left(t-t^{2}\right) \Gamma(3-\theta)}{2+4 \Gamma(3-\theta)} \int_{0}^{1} \frac{(1-\tau)^{\nu-3}}{\Gamma(v-2)} g(\tau) \mathrm{d} \tau \\
& \left.+\frac{\left(t-t^{2}\right) \Gamma(3-\theta)}{2+4 \Gamma(3-\theta)} \int_{0}^{1} \frac{(1-\tau)^{\nu-\theta-1}}{\Gamma(v-\theta)} g(\tau) \mathrm{d} \tau\right] .
\end{aligned}
$$

Proof Let $\varrho_{0}$ be a solution for hybrid equation (5). Then the general solution of homogeneous equation (5) is obtained by the equality $\frac{\varrho_{0}(t)}{w\left(t, \varrho_{0}(t)\right)}=\mathcal{I}^{v} g(t)+b_{0}^{*}+b_{1}^{*} t+b_{2}^{*} t^{2}$, where $b_{0}^{*}, b_{1}^{*}, b_{2}^{*} \in \mathbb{R}$. That is,

$$
\varrho_{0}(t)=w\left(t, \varrho_{0}(t)\right)\left[\int_{0}^{t} \frac{(t-\tau)^{\nu-1}}{\Gamma(v)} g(\tau) \mathrm{d} \tau+b_{0}^{*}+b_{1}^{*} t+b_{2}^{*} t^{2}\right] .
$$

Now, we employ the Caputo derivative of arbitrary orders on both sides of equation (8), and we get

$$
\begin{aligned}
& \mathcal{D}\left(\frac{\varrho_{0}(t)}{w\left(t, \varrho_{0}(t)\right)}\right)=\int_{0}^{t} \frac{(t-\tau)^{\nu-2}}{\Gamma(v-1)} g(\tau) \mathrm{d} \tau+b_{1}^{*}+2 b_{2}^{*} t, \\
& \mathcal{D}^{2}\left(\frac{\varrho_{0}(t)}{w\left(t, \varrho_{0}(t)\right)}\right)=\int_{0}^{t} \frac{(t-\tau)^{\nu-3}}{\Gamma(v-2)} g(\tau) \mathrm{d} \tau+2 b_{2}^{*}, \\
& { }^{c} \mathcal{D}^{\theta}\left(\frac{\varrho_{0}(t)}{w\left(t, \varrho_{0}(t)\right)}\right)=\int_{0}^{t} \frac{(t-\tau)^{\nu-\theta-1}}{\Gamma(v-\theta)} g(\tau) \mathrm{d} \tau+b_{2}^{*} \frac{2 t^{2-\theta}}{\Gamma(3-\theta)},
\end{aligned}
$$

where $1<\theta \leq 2$. Corresponding to the boundary value conditions, we obtain $b_{0}^{*}=0$ and

$$
\begin{aligned}
b_{1}^{*}= & -\frac{1}{3} \int_{0}^{1} \frac{(1-\tau)^{\nu-1}}{\Gamma(\nu)} g(\tau) \mathrm{d} \tau-\frac{1}{3} \int_{0}^{1} \frac{(1-\tau)^{\nu-2}}{\Gamma(\nu-1)} g(\tau) \mathrm{d} \tau \\
& +\frac{\Gamma(3-\theta)}{2+4 \Gamma(3-\theta)} \int_{0}^{1} \frac{(1-\tau)^{\nu-3}}{\Gamma(\nu-2)} g(\tau) \mathrm{d} \tau \\
& +\frac{\Gamma(3-\theta)}{2+4 \Gamma(3-\theta)} \int_{0}^{1} \frac{(1-\tau)^{\nu-\theta-1}}{\Gamma(\nu-\theta)} g(\tau) \mathrm{d} \tau
\end{aligned}
$$

and $b_{2}^{*}=\frac{-\Gamma(3-\theta)}{2+4 \Gamma(3-\theta)} \int_{0}^{1} \frac{(1-\tau)^{\nu-3}}{\Gamma(\nu-2)} g(\tau) \mathrm{d} \tau-\frac{\Gamma(3-\theta)}{2+4 \Gamma(3-\theta)} \int_{0}^{1} \frac{(1-\tau)^{\nu-\theta-1}}{\Gamma(\nu-\theta)} g(\tau) \mathrm{d} \tau$. By putting the values $b_{0}^{*}$, $b_{1}^{*}$, and $b_{2}^{*}$ in equation (8), we have

$$
\begin{aligned}
\varrho_{0}(t)= & w\left(t, \varrho_{0}(t)\right)\left[\int_{0}^{t} \frac{(t-\tau)^{\nu-1}}{\Gamma(\nu)} g(\tau) \mathrm{d} \tau-\frac{t}{3} \int_{0}^{1} \frac{(1-\tau)^{\nu-1}}{\Gamma(\nu)} g(\tau) \mathrm{d} \tau\right. \\
& -\frac{t}{3} \int_{0}^{1} \frac{(1-\tau)^{\nu-2}}{\Gamma(v-1)} g(\tau) \mathrm{d} \tau+\frac{\left(t-t^{2}\right) \Gamma(3-\theta)}{2+4 \Gamma(3-\theta)} \int_{0}^{1} \frac{(1-\tau)^{\nu-3}}{\Gamma(v-2)} g(\tau) \mathrm{d} \tau \\
& \left.+\frac{\left(t-t^{2}\right) \Gamma(3-\theta)}{2+4 \Gamma(3-\theta)} \int_{0}^{1} \frac{(1-\tau)^{\nu-\theta-1}}{\Gamma(v-\theta)} g(\tau) \mathrm{d} \tau\right] .
\end{aligned}
$$

This means that $\varrho_{0}$ is a solution for integral equation (7). On the contrary, it is easy to check that $\varrho_{0}$ satisfies the fractional hybrid BVP (5)-(6) if $\varrho_{0}$ is a solution for the integral equation of fractional order (7). 
Theorem 5 Suppose that $w$ is a nonzero continuous real-valued function on $[0,1] \times \mathbb{R}$ and $\Phi \in C_{\mathbb{R}}([0,1] \times \mathbb{R})$. Furthermore, assume that the following statements hold:

$(\mathcal{A} 1)$ There exists a bounded real-valued map $s:[0,1] \rightarrow \mathbb{R}^{+}$so that $\left|w\left(t, \varrho_{1}\right)-w\left(t, \varrho_{1}^{*}\right)\right| \leq$ $s(t)\left|\varrho_{1}-\varrho_{1}^{*}\right|$ for all $\varrho_{1}, \varrho_{1}^{*} \in \mathbb{R}$

$(\mathcal{A} 2)$ There exist a nondecreasing and continuous map $\xi: \mathbb{R}^{\geq 0} \rightarrow \mathbb{R}^{+}$and a continuous map $h:[0,1] \rightarrow \mathbb{R}^{+}$provided that $|\Phi(t, \varrho)| \leq h(t) \xi(\|\varrho\|)$ for each $\varrho \in \mathbb{R}$ and $t \in$ $[0,1]$

(A3) There exists a number $\varepsilon \in \mathbb{R}^{+}$so that

$$
\varepsilon>\frac{W^{*} \Delta^{*} H^{*} \xi(\|\varrho\|)}{1-s^{*} \Delta^{*} H^{*} \xi(\|\varrho\|)}
$$

where $W^{*}=\sup _{t \in[0,1]}|w(t, 0)|, H^{*}=\sup _{t \in[0,1]}|h(t)|, s^{*}=\sup _{t \in[0,1]}|s(t)|$, and

$$
\begin{aligned}
\Delta^{*}= & \frac{4+v}{3 \Gamma(v+1)} \\
& +\frac{\Gamma(3-\theta)}{(2+4 \Gamma(3-\theta)) \Gamma(v-1)}+\frac{\Gamma(3-\theta)}{(2+4 \Gamma(3-\theta)) \Gamma(v-\theta+1)} .
\end{aligned}
$$

If $s^{*} \Delta^{*} H^{*} \xi(\|\varrho\|)<1$, then the hybrid fractional problem (1)-(2) has a solution on $[0,1]$.

Proof We construct the closed ball $\overline{\mathcal{V}}_{\varepsilon}(0):=\left\{\varrho \in \mathcal{X}:\|\varrho\|_{\mathcal{X}} \leq \varepsilon\right\}$, where $\varepsilon$ satisfies inequality (9). Consider the operators $\Upsilon_{1}, \Upsilon_{2}: \overline{\mathcal{V}}_{\epsilon}(0) \rightarrow \mathcal{X}$ given by $\left(\Upsilon_{1} \varrho\right)(t)=w(t, \varrho(t))$ and

$$
\begin{aligned}
\left(\Upsilon_{2} \varrho\right)(t)= & \int_{0}^{t} \frac{(t-\tau)^{\nu-1}}{\Gamma(\nu)} \Phi(\tau, \varrho(\tau)) \mathrm{d} \tau-\frac{t}{3} \int_{0}^{1} \frac{(1-\tau)^{\nu-1}}{\Gamma(\nu)} \Phi(\tau, \varrho(\tau)) \mathrm{d} \tau \\
& -\frac{t}{3} \int_{0}^{1} \frac{(1-\tau)^{\nu-2}}{\Gamma(v-1)} \Phi(\tau, \varrho(\tau)) \mathrm{d} \tau \\
& +\frac{\left(t-t^{2}\right) \Gamma(3-\theta)}{2+4 \Gamma(3-\theta)} \int_{0}^{1} \frac{(1-\tau)^{\nu-3}}{\Gamma(v-2)} \Phi(\tau, \varrho(\tau)) \mathrm{d} \tau \\
& +\frac{\left(t-t^{2}\right) \Gamma(3-\theta)}{2+4 \Gamma(3-\theta)} \int_{0}^{1} \frac{(1-\tau)^{\nu-\theta-1}}{\Gamma(v-\theta)} \Phi(\tau, \varrho(\tau)) \mathrm{d} \tau .
\end{aligned}
$$

Obviously, $\varrho \in \mathcal{X}$ as a solution for hybrid BVP (1)-(2) satisfies equation $\Upsilon_{1} \varrho \Upsilon_{2} \varrho=\varrho$. First, we want to show that the operator $\Upsilon_{1}$ is Lipschitzian on $\mathcal{X}$ with Lipschitz constant $s^{*}=\sup _{t \in[0,1]}|s(t)|$. To check this, let $\varrho_{1}, \varrho_{2} \in \overline{\mathcal{V}}_{\varepsilon}(0)$. By using assumption $(\mathcal{A} 1)$, we have $\left|\left(\Upsilon_{1} \varrho_{1}\right)(t)-\left(\Upsilon_{1} \varrho_{2}\right)(t)\right|=\left|w\left(t, \varrho_{1}(t)\right)-w\left(t, \varrho_{2}(t)\right)\right| \leq s(t)\left|\varrho_{1}(t)-\varrho_{2}(t)\right|$ for all $t \in[0,1]$. Hence, $\left\|\Upsilon_{1} \varrho_{1}-\Upsilon_{1} \varrho_{2}\right\|_{\mathcal{X}} \leq s^{*}\left\|\varrho_{1}-\varrho_{2}\right\| \mathcal{X}$ for all $\varrho_{1}, \varrho_{2} \in \overline{\mathcal{V}}_{\varepsilon}(0)$. This inequality shows that $\Upsilon_{1}$ is Lipschitzian on $\overline{\mathcal{V}}_{\varepsilon}(0)$ with constant $s^{*}$. In the sequel, we have to show that $\Upsilon_{2}$ on $\overline{\mathcal{V}}_{\varepsilon}(0)$ is completely continuous. In this way, we need to show the continuity of $\Upsilon_{2}$ on $\overline{\mathcal{V}}_{\varepsilon}(0)$. Consider the convergent sequence $\left\{\varrho_{n}\right\}$ in the ball $\overline{\mathcal{V}}_{\varepsilon}(0)$ with $\varrho_{n} \rightarrow \varrho$, where $\varrho$ is an arbitrary member belonging to $\overline{\mathcal{V}}_{\varepsilon}(0)$. By the hypothesis, we know that $\Phi$ is continuous. Thus we have $\lim _{n \rightarrow \infty} \Phi\left(t, \varrho_{n}(t)\right)=\Phi(t, \varrho(t))$. In view of the dominated convergence theorem due to Lebesgue, we have

$$
\lim _{n \rightarrow \infty}\left(\Upsilon_{2} \varrho_{n}\right)(t)=\int_{0}^{t} \frac{(t-\tau)^{\nu-1}}{\Gamma(v)} \lim _{n \rightarrow \infty} \Phi\left(\tau, \varrho_{n}(\tau)\right) \mathrm{d} \tau
$$




$$
\begin{aligned}
& -\frac{t}{3} \int_{0}^{1} \frac{(1-\tau)^{\nu-1}}{\Gamma(\nu)} \lim _{n \rightarrow \infty} \Phi\left(\tau, \varrho_{n}(\tau)\right) \mathrm{d} \tau \\
& -\frac{t}{3} \int_{0}^{1} \frac{(1-\tau)^{\nu-2}}{\Gamma(\nu-1)} \lim _{n \rightarrow \infty} \Phi\left(\tau, \varrho_{n}(\tau)\right) \mathrm{d} \tau \\
& +\frac{\left(t-t^{2}\right) \Gamma(3-\theta)}{2+4 \Gamma(3-\theta)} \int_{0}^{1} \frac{(1-\tau)^{\nu-3}}{\Gamma(v-2)} \lim _{n \rightarrow \infty} \Phi\left(\tau, \varrho_{n}(\tau)\right) \mathrm{d} \tau \\
& +\frac{\left(t-t^{2}\right) \Gamma(3-\theta)}{2+4 \Gamma(3-\theta)} \int_{0}^{1} \frac{(1-\tau)^{\nu-\theta-1}}{\Gamma(\nu-\theta)} \lim _{n \rightarrow \infty} \Phi\left(\tau, \varrho_{n}(\tau)\right) \mathrm{d} \tau \\
& =\int_{0}^{t} \frac{(t-\tau)^{\nu-1}}{\Gamma(\nu)} \int^{1} \frac{(1-\tau)^{\nu-1}}{\Gamma(\nu)} \Phi(\tau, \varrho(\tau)) \mathrm{d} \tau \\
& -\frac{t}{3} \int_{0}^{1} \frac{(1-\tau)^{\nu-2}}{\Gamma(\nu-1)} \Phi(\tau, \varrho(\tau)) \mathrm{d} \tau \\
& +\frac{\left(t-t^{2}\right) \Gamma(3-\theta)}{2+4 \Gamma(3-\theta)} \int_{0}^{1} \frac{(1-\tau)^{\nu-3}}{\Gamma(\nu-2)} \Phi(\tau, \varrho(\tau)) \mathrm{d} \tau \\
& +\frac{\left(t-t^{2}\right) \Gamma(3-\theta)}{2+4 \Gamma(3-\theta)} \int_{0}^{1} \frac{(1-\tau)^{\nu-\theta-1}}{\Gamma(\nu-\theta)} \Phi(\tau, \varrho(\tau)) \mathrm{d} \tau \\
& =\left(\Upsilon_{2} \varrho\right)(t)
\end{aligned}
$$

for any $t \in[0,1]$. Therefore, $\Upsilon_{2} \varrho_{n} \rightarrow \Upsilon_{2} \varrho$, and so we deduce that $\Upsilon_{2}$ is continuous on $\overline{\mathcal{V}}_{\varepsilon}(0)$. Now, we check the uniform boundedness of $\Upsilon_{2}$ on $\overline{\mathcal{V}}_{\varepsilon}(0)$. By using assumption $(\mathcal{A} 2)$, we can write

$$
\begin{aligned}
\left|\left(\Upsilon_{2} \varrho\right)(t)\right|= & \int_{0}^{t} \frac{(t-\tau)^{\nu-1}}{\Gamma(\nu)}|\Phi(\tau, \varrho(\tau))| \mathrm{d} \tau+\frac{t}{3} \int_{0}^{1} \frac{(1-\tau)^{\nu-1}}{\Gamma(\nu)}|\Phi(\tau, \varrho(\tau))| \mathrm{d} \tau \\
& +\frac{t}{3} \int_{0}^{1} \frac{(1-\tau)^{\nu-2}}{\Gamma(v-1)}|\Phi(\tau, \varrho(\tau))| \mathrm{d} \tau \\
& +\frac{\left|t-t^{2}\right| \Gamma(3-\theta)}{2+4 \Gamma(3-\theta)} \int_{0}^{1} \frac{(1-\tau)^{\nu-3}}{\Gamma(v-2)}|\Phi(\tau, \varrho(\tau))| \mathrm{d} \tau \\
& +\frac{\left|t-t^{2}\right| \Gamma(3-\theta)}{2+4 \Gamma(3-\theta)} \int_{0}^{1} \frac{(1-\tau)^{\nu-\theta-1}}{\Gamma(v-\theta)}|\Phi(\tau, \varrho(\tau))| \mathrm{d} \tau \\
\leq & \frac{t^{\nu}}{\Gamma(\nu+1)} h(t) \xi(\|\varrho\|)+\frac{t}{3 \Gamma(v+1)} h(t) \xi(\|\varrho\|)+\frac{t}{3 \Gamma(\nu)} h(t) \xi(\|\varrho\|) \\
& +\frac{\left|t-t^{2}\right| \Gamma(3-\theta)}{(2+4 \Gamma(3-\theta)) \Gamma(v-1)} h(t) \xi(\|\varrho\|) \\
& +\frac{\left|t-t^{2}\right| \Gamma(3-\theta)}{(2+4 \Gamma(3-\theta)) \Gamma(\nu-\theta+1)} h(t) \xi(\|\varrho\|)
\end{aligned}
$$

for any $t \in[0,1]$ and $\varrho \in \overline{\mathcal{V}}_{\varepsilon}(0)$. Hence $\left\|\Upsilon_{2} \varrho\right\|_{\mathcal{X}} \leq H^{*} \xi(\|\varrho\|) \Delta^{*}$, where $\Delta^{*}$ is defined in (10). This means that $\Upsilon_{2}\left(\overline{\mathcal{V}}_{\varepsilon}(0)\right)$ is a uniformly bounded subset of $\mathcal{X}$. Here, we proceed to proving that the operator $\Upsilon_{2}$ is equicontinuous. Without loss of generality, let us assume that $0 \leq t_{1}, t_{2} \leq 1$ provided that $t_{1}<t_{2}$, and let $\varrho \in \overline{\mathcal{V}}_{\varepsilon}(0)$. Then

$$
\begin{aligned}
& \left|\left(\Upsilon_{2} \varrho\right)\left(t_{2}\right)-\left(\Upsilon_{2} \varrho\right)\left(t_{1}\right)\right| \\
& \quad=\left|\int_{0}^{t_{2}} \frac{\left(t_{2}-\tau\right)^{\nu-1}}{\Gamma(\nu)} \Phi(\tau, \varrho(\tau)) \mathrm{d} \tau-\int_{0}^{t_{1}} \frac{\left(t_{1}-\tau\right)^{\nu-1}}{\Gamma(\nu)} \Phi(\tau, \varrho(\tau)) \mathrm{d} \tau\right|
\end{aligned}
$$




$$
\begin{aligned}
& +\frac{\left|t_{2}-t_{1}\right|}{3} \int_{0}^{1} \frac{(1-\tau)^{\nu-1}}{\Gamma(v)}|\Phi(\tau, \varrho(\tau))| \mathrm{d} \tau \\
& +\frac{\left|t_{2}-t_{1}\right|}{3} \int_{0}^{1} \frac{(1-\tau)^{\nu-2}}{\Gamma(v-1)}|\Phi(\tau, \varrho(\tau))| \mathrm{d} \tau \\
& +\frac{\left|\left(t_{2}-t_{1}\right)-\left(t_{2}-t_{1}\right)^{2}\right| \Gamma(3-\theta)}{2+4 \Gamma(3-\theta)} \int_{0}^{1} \frac{(1-\tau)^{\nu-3}}{\Gamma(v-2)}|\Phi(\tau, \varrho(\tau))| \mathrm{d} \tau \\
& +\frac{\left|\left(t_{2}-t_{1}\right)-\left(t_{2}-t_{1}\right)^{2}\right| \Gamma(3-\theta)}{2+4 \Gamma(3-\theta)} \int_{0}^{1} \frac{(1-\tau)^{\nu-\theta-1}}{\Gamma(\nu-\theta)}|\Phi(\tau, \varrho(\tau))| \mathrm{d} \tau .
\end{aligned}
$$

Letting $t_{1} \rightarrow t_{2}$, we observe that the right-hand side of the above inequality converges to zero independently of $\varrho \in \overline{\mathcal{V}}_{\varepsilon}(0)$. Thus $\left|\left(\Upsilon_{2} \varrho\right)\left(t_{2}\right)-\left(\Upsilon_{2} \varrho\right)\left(t_{1}\right)\right| \rightarrow 0$ as $t_{1} \rightarrow t_{2}$. Consequently, the operator $\Upsilon_{2}$ is equicontinuous. By utilizing the Arzela-Ascoli theorem, we arrive at the desired aim, which is the complete continuity of $\Upsilon_{2}$ on $\overline{\mathcal{V}}_{\varepsilon}(0)$. In addition, because of hypothesis $(\mathcal{A} 3)$, we get

$$
\begin{aligned}
\mathcal{K}_{0}^{*}= & \left\|\Upsilon_{2}\left(\overline{\mathcal{V}}_{\varepsilon}(0)\right)\right\|_{\mathcal{X}} \\
= & \sup _{t \in[0,1]}\left\{\left|\left(\Upsilon_{2} \varrho\right)(t)\right|: \varrho \in \overline{\mathcal{V}}_{\varepsilon}(0)\right\}=H^{*} \xi(\|\varrho\|) \\
& \times\left[\frac{4+v}{3 \Gamma(v+1)}+\frac{\Gamma(3-\theta)}{(2+4 \Gamma(3-\theta)) \Gamma(v-1)}+\frac{\Gamma(3-\theta)}{(2+4 \Gamma(3-\theta)) \Gamma(v-\theta+1)}\right] \\
= & H^{*} \xi(\|\varrho\|) \Delta^{*} .
\end{aligned}
$$

Setting $l^{*}=s^{*}$, we get $l^{*} H^{*}<1$. Now, to complete the proof, we claim that one of conditions $(b 1)$ or $(b 2)$ in Theorem 1 is valid. Let $\varrho=\mu \Upsilon_{1} \varrho \Upsilon_{2} \varrho$ for some constant $0<\mu<1$ and $\|\varrho\|=\varepsilon$. Then we have the following estimate:

$$
\begin{aligned}
|\varrho(t)|= & \mu\left|\left(\Upsilon_{1} \varrho\right)(t)\right|\left|\left(\Upsilon_{2} \varrho\right)(t)\right| \\
= & \mu|w(t, \varrho(t))| \\
& \times \mid \int_{0}^{t} \frac{(t-\tau)^{\nu-1}}{\Gamma(v)} \Phi(\tau, \varrho(\tau)) \mathrm{d} \tau-\frac{t}{3} \int_{0}^{1} \frac{(1-\tau)^{\nu-1}}{\Gamma(v)} \Phi(\tau, \varrho(\tau)) \mathrm{d} \tau \\
& -\frac{t}{3} \int_{0}^{1} \frac{(1-\tau)^{\nu-2}}{\Gamma(v-1)} \Phi(\tau, \varrho(\tau)) \mathrm{d} \tau \\
& +\frac{\left(t-t^{2}\right) \Gamma(3-\theta)}{2+4 \Gamma(3-\theta)} \int_{0}^{1} \frac{(1-\tau)^{\nu-3}}{\Gamma(v-2)} \Phi(\tau, \varrho(\tau)) \mathrm{d} \tau \\
& +\frac{\left(t-t^{2}\right) \Gamma(3-\theta)}{2+4 \Gamma(3-\theta)} \int_{0}^{1} \frac{(1-\tau)^{\nu-\theta-1}}{\Gamma(v-\theta)} \Phi(\tau, \varrho(\tau)) \mathrm{d} \tau \mid \\
\leq & (|w(t, \varrho(t))-w(t, 0)|+|w(t, 0)|) \\
& \times\left(\int_{0}^{t} \frac{(t-\tau)^{\nu-1}}{\Gamma(v)}|\Phi(\tau, \varrho(\tau))| \mathrm{d} \tau+\frac{t}{3} \int_{0}^{1} \frac{(1-\tau)^{\nu-1}}{\Gamma(\nu)}|\Phi(\tau, \varrho(\tau))| \mathrm{d} \tau\right. \\
& +\frac{t}{3} \int_{0}^{1} \frac{(1-\tau)^{\nu-2}}{\Gamma(v-1)}|\Phi(\tau, \varrho(\tau))| \mathrm{d} \tau+\frac{\left|t-t^{2}\right| \Gamma(3-\theta)}{2+4 \Gamma(3-\theta)} \\
& \times \int_{0}^{1} \frac{(1-\tau)^{\nu-3}}{\Gamma(v-2)}|\Phi(\tau, \varrho(\tau))| \mathrm{d} \tau
\end{aligned}
$$




$$
\begin{aligned}
& \left.+\frac{\left|t-t^{2}\right| \Gamma(3-\theta)}{2+4 \Gamma(3-\theta)} \int_{0}^{1} \frac{(1-\tau)^{\nu-\theta-1}}{\Gamma(v-\theta)}|\Phi(\tau, \varrho(\tau))| \mathrm{d} \tau\right) \\
\leq & \left(s(t)|\varrho(t)|+W^{*}\right) \Delta^{*} H^{*} \xi(\|\varrho\|) \\
\leq & \left(s^{*}\|\varrho\|+W^{*}\right) \Delta^{*} H^{*} \xi(\|\varrho\|),
\end{aligned}
$$

and so $\varepsilon \leq \frac{W^{*} \Delta^{*} H^{*} \xi(\|\varrho\|)}{1-s^{*} \Delta^{*} H^{*} \xi(\|\varrho\|)}$, which is a contradiction to inequality (9). It follows that condition $(b 1)$ of Theorem 1 is not possible. Hence, condition $(b 2)$ is valid and so the mentioned fractional hybrid problem (1)-(2) has a solution.

In what follows, we are going to provide another essential result for the fractional hybrid inclusion problem (3)-(4). Existence results herein are carried out in light of the assumptions of Theorem 3.

Definition 6 We say that the function $\varrho \in A C_{\mathbb{R}}([0,1])$ is a solution for the hybrid inclusion BVP of fractional order (3)-(4) whenever there exists an integrable function $\vartheta \in \mathcal{L}_{\mathbb{R}}^{1}([0,1])$ with $\vartheta(t) \in \Psi(t, \varrho(t))$ for almost all $t \in[0,1]$ satisfying $\left.\left(\frac{\varrho(t)}{w(t, \varrho(t))}\right)\right|_{t=0}=0$ and

$$
\begin{aligned}
& \left.\mathcal{D}\left(\frac{\varrho(t)}{w(t, \varrho(t))}\right)\right|_{t=0}=-\left.\left(\frac{\varrho(t)}{w(t, \varrho(t))}\right)\right|_{t=1}-\left.\mathcal{D}\left(\frac{\varrho(t)}{w(t, \varrho(t))}\right)\right|_{t=1}, \\
& \left.\mathcal{D}^{2}\left(\frac{\varrho(t)}{w(t, \varrho(t))}\right)\right|_{t=0}=-\left.\mathcal{D}^{2}\left(\frac{\varrho(t)}{w(t, \varrho(t))}\right)\right|_{t=1}-\left.{ }^{c} \mathcal{D}^{\theta}\left(\frac{\varrho(t)}{w(t, \varrho(t))}\right)\right|_{t=1}
\end{aligned}
$$

and

$$
\begin{aligned}
\varrho(t)= & w(t, \varrho(t))\left[\int_{0}^{t} \frac{(t-\tau)^{\nu-1}}{\Gamma(\nu)} \vartheta(\tau) \mathrm{d} \tau-\frac{t}{3} \int_{0}^{1} \frac{(1-\tau)^{\nu-1}}{\Gamma(\nu)} \vartheta(\tau) \mathrm{d} \tau\right. \\
& -\frac{t}{3} \int_{0}^{1} \frac{(1-\tau)^{\nu-2}}{\Gamma(v-1)} \vartheta(\tau) \mathrm{d} \tau+\frac{\left(t-t^{2}\right) \Gamma(3-\theta)}{2+4 \Gamma(3-\theta)} \int_{0}^{1} \frac{(1-\tau)^{\nu-3}}{\Gamma(\nu-2)} \vartheta(\tau) \mathrm{d} \tau \\
& \left.+\frac{\left(t-t^{2}\right) \Gamma(3-\theta)}{2+4 \Gamma(3-\theta)} \int_{0}^{1} \frac{(1-\tau)^{\nu-\theta-1}}{\Gamma(\nu-\theta)} \vartheta(\tau) \mathrm{d} \tau\right]
\end{aligned}
$$

for each $t \in[0,1]$.

Here, we can formulate desired theorem on the existence of solution function of the above form.

\section{Theorem 7 Assume that the following statements are valid:}

$\left(\mathcal{A}^{\prime} 1\right)$ There is a bounded real-valued function $s:[0,1] \rightarrow \mathbb{R}^{+}$such that $\mid w\left(t, \varrho_{1}(t)\right)$ $w\left(t, \varrho_{2}(t)\right)|\leq s(t)| \varrho_{1}(t)-\varrho_{2}(t) \mid$ for all $\varrho_{1}, \varrho_{2} \in \mathbb{R}$ and $t \in[0,1] ;$

$\left(\mathcal{A}^{\prime} 2\right)$ The convex and compact-valued multifunction $\Psi:[0,1] \times \mathbb{R} \rightarrow \mathcal{P}_{c p, c v}(\mathbb{R})$ has an $\mathcal{L}^{1}$-Caratheodory property;

$\left(\mathcal{A}^{\prime} 3\right)$ There is a map $\sigma \in \mathcal{L}_{\mathbb{R}^{+}}^{1}([0,1])$ such that $\|\Psi(t, \varrho)\|=\sup \{|\vartheta|: \vartheta \in \Psi(t, \varrho(t))\} \leq \sigma(t)$ for any $\varrho \in \mathbb{R}$ and almost all $t \in[0,1]$. Here, $\|\sigma\|_{\mathcal{L}^{1}}=\int_{0}^{1}|\sigma(\tau)| \mathrm{d} \tau ;$

( $\left.\mathcal{A}^{\prime} 4\right)$ There is a number $\tilde{\varepsilon} \in \mathbb{R}^{+}$such that

$$
\tilde{\varepsilon}>\frac{W^{*} \Delta^{*}\|\sigma\|_{\mathcal{L}^{1}}}{1-s^{*} \Delta^{*}\|\sigma\|_{\mathcal{L}^{1}}}
$$

where $W^{*}=\sup _{t \in[0,1]}|w(t, 0)|, s^{*}=\sup _{t \in[0,1]}|s(t)|$ and $\Delta^{*}$ is given in $(10)$. 


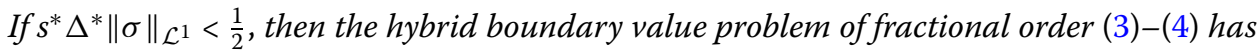
a solution.

Proof To transform the hybrid inclusion problem (3)-(4) into a fixed point problem, we define $\mathcal{K}: \mathcal{X} \rightarrow \mathcal{P}(\mathcal{X})$ by $\mathcal{K}(\varrho)=\left\{\delta \in \mathcal{X}: \delta(t)=v_{\vartheta}(t) t \in[0,1]\right\}$, where $\vartheta \in(\mathcal{S E} \mathcal{L})_{\Psi, \varrho}$ and

$$
\begin{aligned}
\nu_{\vartheta}(t)= & w(t, \varrho(t))\left(\int_{0}^{t} \frac{(t-\tau)^{\nu-1}}{\Gamma(\nu)} \vartheta(\tau) \mathrm{d} \tau-\frac{t}{3} \int_{0}^{1} \frac{(1-\tau)^{\nu-1}}{\Gamma(\nu)} \vartheta(\tau) \mathrm{d} \tau\right. \\
& -\frac{t}{3} \int_{0}^{1} \frac{(1-\tau)^{\nu-2}}{\Gamma(v-1)} \vartheta(\tau) \mathrm{d} \tau+\frac{\left(t-t^{2}\right) \Gamma(3-\theta)}{2+4 \Gamma(3-\theta)} \int_{0}^{1} \frac{(1-\tau)^{\nu-3}}{\Gamma(v-2)} \vartheta(\tau) \mathrm{d} \tau \\
& \left.+\frac{\left(t-t^{2}\right) \Gamma(3-\theta)}{2+4 \Gamma(3-\theta)} \int_{0}^{1} \frac{(1-\tau)^{\nu-\theta-1}}{\Gamma(v-\theta)} \vartheta(\tau) \mathrm{d} \tau\right) .
\end{aligned}
$$

It is clear that each fixed point of $\mathcal{K}$ is a solution for hybrid inclusion BVP (3)-(4). We split the operator $\mathcal{K}$ into two parts as $\Upsilon_{1}: \mathcal{X} \rightarrow \mathcal{X}$ and $\Upsilon_{2}: \mathcal{X} \rightarrow \mathcal{P}(\mathcal{X})$ defined by $\left(\Upsilon_{1} \varrho\right)(t)=$ $w(t, \varrho(t))$ and $\left(\Upsilon_{2} \varrho\right)(t)=\left\{\zeta \in \mathcal{X}: \zeta(t)=\phi_{\vartheta}(t), t \in[0,1]\right\}$, where $\vartheta \in(\mathcal{S E L})_{\Psi, \varrho}$ and

$$
\begin{aligned}
\phi_{\vartheta} & (t) \int_{0}^{t} \frac{(t-\tau)^{\nu-1}}{\Gamma(\nu)} \vartheta(\tau) \mathrm{d} \tau-\frac{t}{3} \int_{0}^{1} \frac{(1-\tau)^{\nu-1}}{\Gamma(\nu)} \vartheta(\tau) \mathrm{d} \tau \\
& -\frac{t}{3} \int_{0}^{1} \frac{(1-\tau)^{\nu-2}}{\Gamma(v-1)} \vartheta(\tau) \mathrm{d} \tau+\frac{\left(t-t^{2}\right) \Gamma(3-\theta)}{2+4 \Gamma(3-\theta)} \int_{0}^{1} \frac{(1-\tau)^{\nu-3}}{\Gamma(\nu-2)} \vartheta(\tau) \mathrm{d} \tau \\
& +\frac{\left(t-t^{2}\right) \Gamma(3-\theta)}{2+4 \Gamma(3-\theta)} \int_{0}^{1} \frac{(1-\tau)^{\nu-\theta-1}}{\Gamma(\nu-\theta)} \vartheta(\tau) \mathrm{d} \tau .
\end{aligned}
$$

Note that $\mathcal{K}(\varrho)=\Upsilon_{1} \varrho \Upsilon_{2} \varrho$. We are going to prove that operators $\Upsilon_{1}$ and $\Upsilon_{2}$ satisfy all the assumptions of Theorem 3. Obviously, in view of hypothesis $\left(\mathcal{A}^{\prime} 1\right)$ and in a similar way used in Theorem 5, one can easily find that $\Upsilon_{1}$ is Lipschitzian on $\mathcal{X}$. In the following, we need to show that $\Upsilon_{2}$ is convex-valued. To do this, let $\varrho_{1}, \varrho_{2} \in \Upsilon_{2} \varrho$. Choose $\vartheta_{1}, \vartheta_{2} \in$ $(\mathcal{S E} \mathcal{L})_{\Psi, \varrho}$ so that

$$
\begin{aligned}
\varrho_{j}(t)= & \int_{0}^{t} \frac{(t-\tau)^{\nu-1}}{\Gamma(v)} \vartheta_{j}(\tau) \mathrm{d} \tau-\frac{t}{3} \int_{0}^{1} \frac{(1-\tau)^{\nu-1}}{\Gamma(v)} \vartheta_{j}(\tau) \mathrm{d} \tau \\
& -\frac{t}{3} \int_{0}^{1} \frac{(1-\tau)^{\nu-2}}{\Gamma(v-1)} \vartheta_{j}(\tau) \mathrm{d} \tau+\frac{\left(t-t^{2}\right) \Gamma(3-\theta)}{2+4 \Gamma(3-\theta)} \int_{0}^{1} \frac{(1-\tau)^{\nu-3}}{\Gamma(\nu-2)} \vartheta_{j}(\tau) \mathrm{d} \tau \\
& +\frac{\left(t-t^{2}\right) \Gamma(3-\theta)}{2+4 \Gamma(3-\theta)} \int_{0}^{1} \frac{(1-\tau)^{\nu-\theta-1}}{\Gamma(\nu-\theta)} \vartheta_{j}(\tau) \mathrm{d} \tau
\end{aligned}
$$

for all $t \in[0,1]$ (a.e.) and $j=1,2$. For any $0<\gamma<1$, we obtain

$$
\begin{aligned}
\gamma \varrho_{1}(t)+(1-\gamma) \varrho_{2}(t)= & \int_{0}^{t} \frac{(t-\tau)^{\nu-1}}{\Gamma(v)}\left[\gamma \vartheta_{1}(\tau)+(1-\gamma) \vartheta_{2}(\tau)\right] \mathrm{d} \tau \\
& -\frac{t}{3} \int_{0}^{1} \frac{(1-\tau)^{\nu-1}}{\Gamma(v)}\left[\gamma \vartheta_{1}(\tau)+(1-\gamma) \vartheta_{2}(\tau)\right] \mathrm{d} \tau \\
& -\frac{t}{3} \int_{0}^{1} \frac{(1-\tau)^{\nu-2}}{\Gamma(\nu-1)}\left[\gamma \vartheta_{1}(\tau)+(1-\gamma) \vartheta_{2}(\tau)\right] \mathrm{d} \tau \\
& +\frac{\left(t-t^{2}\right) \Gamma(3-\theta)}{2+4 \Gamma(3-\theta)} \int_{0}^{1} \frac{(1-\tau)^{\nu-3}}{\Gamma(v-2)}\left[\gamma \vartheta_{1}(\tau)+(1-\gamma) \vartheta_{2}(\tau)\right] \mathrm{d} \tau
\end{aligned}
$$




$$
+\frac{\left(t-t^{2}\right) \Gamma(3-\theta)}{2+4 \Gamma(3-\theta)} \int_{0}^{1} \frac{(1-\tau)^{\nu-\theta-1}}{\Gamma(\nu-\theta)}\left[\gamma \vartheta_{1}(\tau)+(1-\gamma) \vartheta_{2}(\tau)\right] \mathrm{d} \tau
$$

for almost all $t \in[0,1]$. By the hypothesis of theorem, $\Psi$ has convex values. Thus $(\mathcal{S E} \mathcal{L})_{\Psi, \varrho}$ is convex-valued and $\gamma \vartheta_{1}(t)+(1-\gamma) \vartheta_{2}(t) \in(\mathcal{S E L})_{\Psi, \varrho}$ for all $t \in[0,1]$ and so $\Upsilon_{2} \varrho$ is a convex set for all $\varrho \in \mathcal{X}$. Now, to check the complete continuity of $\Upsilon_{2}$, we have to prove that $\Upsilon_{2}(\mathcal{X})$ is uniformly bounded and equicontinuous. To reach this purpose, we show that $\Upsilon_{2}$ maps all bounded sets into bounded subsets of the space $\mathcal{X}$. For a number $\varepsilon^{*} \in \mathbb{R}^{+}$, we construct a bounded ball $V_{\varepsilon^{*}}=\left\{\varrho \in \mathcal{X}:\|\varrho\|_{\mathcal{X}} \leq \varepsilon^{*}\right\}$. For every $\varrho \in V_{\varepsilon^{*}}$ and $\zeta \in \Upsilon_{2} \varrho$, there exists $\vartheta \in(\mathcal{S E L})_{\Psi, \varrho}$ so that

$$
\begin{aligned}
\zeta(t)= & \int_{0}^{t} \frac{(t-\tau)^{\nu-1}}{\Gamma(\nu)} \vartheta(\tau) \mathrm{d} \tau-\frac{t}{3} \int_{0}^{1} \frac{(1-\tau)^{\nu-1}}{\Gamma(\nu)} \vartheta(\tau) \mathrm{d} \tau \\
& -\frac{t}{3} \int_{0}^{1} \frac{(1-\tau)^{\nu-2}}{\Gamma(\nu-1)} \vartheta(\tau) \mathrm{d} \tau+\frac{\left(t-t^{2}\right) \Gamma(3-\theta)}{2+4 \Gamma(3-\theta)} \int_{0}^{1} \frac{(1-\tau)^{\nu-3}}{\Gamma(\nu-2)} \vartheta(\tau) \mathrm{d} \tau \\
& +\frac{\left(t-t^{2}\right) \Gamma(3-\theta)}{2+4 \Gamma(3-\theta)} \int_{0}^{1} \frac{(1-\tau)^{\nu-\theta-1}}{\Gamma(\nu-\theta)} \vartheta(\tau) \mathrm{d} \tau
\end{aligned}
$$

for each $t \in[0,1]$. Then we have the following estimate for function $\zeta$ :

$$
\begin{aligned}
& |\zeta(t)| \leq \int_{0}^{t} \frac{(t-\tau)^{\nu-1}}{\Gamma(\nu)}|\vartheta(\tau)| \mathrm{d} \tau-\frac{t}{3} \int_{0}^{1} \frac{(1-\tau)^{\nu-1}}{\Gamma(\nu)}|\vartheta(\tau)| \mathrm{d} \tau \\
& -\frac{t}{3} \int_{0}^{1} \frac{(1-\tau)^{\nu-2}}{\Gamma(\nu-1)}|\vartheta(\tau)| \mathrm{d} \tau \\
& +\frac{\left(t-t^{2}\right) \Gamma(3-\theta)}{2+4 \Gamma(3-\theta)} \int_{0}^{1} \frac{(1-\tau)^{\nu-3}}{\Gamma(v-2)}|\vartheta(\tau)| \mathrm{d} \tau \\
& +\frac{\left(t-t^{2}\right) \Gamma(3-\theta)}{2+4 \Gamma(3-\theta)} \int_{0}^{1} \frac{(1-\tau)^{\nu-\theta-1}}{\Gamma(\nu-\theta)}|\vartheta(\tau)| \mathrm{d} \tau \\
& \leq \int_{0}^{t} \frac{(t-\tau)^{\nu-1}}{\Gamma(v)} \sigma(\tau) \mathrm{d} \tau-\frac{t}{3} \int_{0}^{1} \frac{(1-\tau)^{\nu-1}}{\Gamma(v)} \sigma(\tau) \mathrm{d} \tau \\
& -\frac{t}{3} \int_{0}^{1} \frac{(1-\tau)^{\nu-2}}{\Gamma(v-1)} \sigma(\tau) \mathrm{d} \tau+\frac{\left(t-t^{2}\right) \Gamma(3-\theta)}{2+4 \Gamma(3-\theta)} \int_{0}^{1} \frac{(1-\tau)^{\nu-3}}{\Gamma(v-2)} \sigma(\tau) \mathrm{d} \tau \\
& +\frac{\left(t-t^{2}\right) \Gamma(3-\theta)}{2+4 \Gamma(3-\theta)} \int_{0}^{1} \frac{(1-\tau)^{\nu-\theta-1}}{\Gamma(\nu-\theta)} \sigma(\tau) \mathrm{d} \tau \\
& \leq\left[\frac{4+v}{3 \Gamma(v+1)}+\frac{\Gamma(3-\theta)}{(2+4 \Gamma(3-\theta)) \Gamma(v-1)}+\frac{\Gamma(3-\theta)}{(2+4 \Gamma(3-\theta)) \Gamma(v-\theta+1)}\right] \\
& \times\|\sigma\|_{\mathcal{L}^{1}}=\Delta^{*}\|\sigma\|_{\mathcal{L}^{1}},
\end{aligned}
$$

where $\Delta^{*}$ is given in (10). Thus, $\|\zeta\| \leq \Delta^{*}\|\sigma\|_{\mathcal{L}^{1}}$ and this means that the set $\Upsilon_{2}(\mathcal{X})$ is uniformly bounded. The next step in this part of the proof is to show that $\Upsilon_{2}$ maps bounded sets into equicontinuous sets. Let $\varrho \in V_{\varepsilon^{*}}$ and $\zeta \in \Upsilon_{2} \varrho$. Choose $\vartheta \in(\mathcal{S E} \mathcal{L})_{\Psi, \varrho}$ provided that

$$
\begin{aligned}
\zeta(t)= & \int_{0}^{t} \frac{(t-\tau)^{\nu-1}}{\Gamma(\nu)} \vartheta(\tau) \mathrm{d} \tau-\frac{t}{3} \int_{0}^{1} \frac{(1-\tau)^{\nu-1}}{\Gamma(\nu)} \vartheta(\tau) \mathrm{d} \tau \\
& -\frac{t}{3} \int_{0}^{1} \frac{(1-\tau)^{\nu-2}}{\Gamma(v-1)} \vartheta(\tau) \mathrm{d} \tau+\frac{\left(t-t^{2}\right) \Gamma(3-\theta)}{2+4 \Gamma(3-\theta)} \int_{0}^{1} \frac{(1-\tau)^{\nu-3}}{\Gamma(\nu-2)} \vartheta(\tau) \mathrm{d} \tau
\end{aligned}
$$




$$
+\frac{\left(t-t^{2}\right) \Gamma(3-\theta)}{2+4 \Gamma(3-\theta)} \int_{0}^{1} \frac{(1-\tau)^{\nu-\theta-1}}{\Gamma(\nu-\theta)} \vartheta(\tau) \mathrm{d} \tau
$$

for all $t \in[0,1]$. We may assume that $0 \leq t_{1}, t_{2} \leq 1$ with $t_{1}<t_{2}$. Then we have

$$
\begin{aligned}
\mid \zeta\left(t_{2}\right) & -\zeta\left(t_{1}\right) \mid \\
\leq & \left|\int_{0}^{t_{2}} \frac{\left(t_{2}-\tau\right)^{\nu-1}}{\Gamma(v)} \vartheta(\tau) \mathrm{d} \tau-\int_{0}^{t_{1}} \frac{\left(t_{1}-\tau\right)^{\nu-1}}{\Gamma(\nu)} \vartheta(\tau) \mathrm{d} \tau\right| \\
& +\frac{\left|t_{2}-t_{1}\right|}{3} \int_{0}^{1} \frac{(1-\tau)^{\nu-1}}{\Gamma(v)}|\vartheta(\tau)| \mathrm{d} \tau \\
& +\frac{\left|t_{2}-t_{1}\right|}{3} \int_{0}^{1} \frac{(1-\tau)^{\nu-2}}{\Gamma(\nu-1)}|\vartheta(\tau)| \mathrm{d} \tau \\
& +\frac{\left|\left(t_{2}-t_{1}\right)-\left(t_{2}-t_{1}\right)^{2}\right| \Gamma(3-\theta)}{2+4 \Gamma(3-\theta)} \int_{0}^{1} \frac{(1-\tau)^{\nu-3}}{\Gamma(\nu-2)}|\vartheta(\tau)| \mathrm{d} \tau \\
& +\frac{\left|\left(t_{2}-t_{1}\right)-\left(t_{2}-t_{1}\right)^{2}\right| \Gamma(3-\theta)}{2+4 \Gamma(3-\theta)} \int_{0}^{1} \frac{(1-\tau)^{\nu-\theta-1}}{\Gamma(\nu-\theta)}|\vartheta(\tau)| \mathrm{d} \tau \\
\leq & \int_{0}^{t_{1}}\left(\frac{\left[\left(t_{2}-\tau\right)^{\nu-1}-\left(t_{1}-\tau\right)^{\nu-1}\right]}{\Gamma(\nu)}\right) \sigma(\tau) \mathrm{d} \tau+\int_{t_{1}}^{t_{2}} \frac{\left(t_{2}-\tau\right)^{\nu-1}}{\Gamma(\nu)} \sigma(\tau) \mathrm{d} \tau \\
& +\frac{\left|t_{2}-t_{1}\right|}{3} \int_{0}^{1} \frac{(1-\tau)^{\nu-1}}{\Gamma(\nu)} \sigma(\tau) \mathrm{d} \tau+\frac{\left|t_{2}-t_{1}\right|}{3} \int_{0}^{1} \frac{(1-\tau)^{\nu-2}}{\Gamma(\nu-1)} \sigma(\tau) \mathrm{d} \tau \\
& +\frac{\left|\left(t_{2}-t_{1}\right)-\left(t_{2}-t_{1}\right)^{2}\right| \Gamma(3-\theta)}{2+4 \Gamma(3-\theta)} \int_{0}^{1} \frac{(1-\tau)^{\nu-3}}{\Gamma(\nu-2)} \sigma(\tau) \mathrm{d} \tau \\
& +\frac{\left|\left(t_{2}-t_{1}\right)-\left(t_{2}-t_{1}\right)^{2}\right| \Gamma(3-\theta)}{2+4 \Gamma(3-\theta)} \int_{0}^{1} \frac{(1-\tau)^{\nu-\theta-1}}{\Gamma(\nu-\theta)} \sigma(\tau) \mathrm{d} \tau .
\end{aligned}
$$

Letting $t_{1} \rightarrow t_{2}$, one can see that the above inequality converges to 0 independently of $\varrho \in V_{\varepsilon^{*}}$. It follows from the Arzela-Ascoli theorem that $\Upsilon_{2}: C_{\mathbb{R}}([0,1]) \rightarrow \mathcal{P}\left(C_{\mathbb{R}}([0,1])\right)$ is a completely continuous operator. Here, we claim that $\Upsilon_{2}$ has a closed graph. Then, because of the complete continuity of $\Upsilon_{2}$, we find that $\Upsilon_{2}$ is upper semi-continuous. For this aim, suppose that $\varrho_{n} \in V_{\varepsilon^{*}}$ and $\zeta_{n} \in \Upsilon_{2} \varrho_{n}$ are such that $\varrho_{n} \rightarrow \varrho^{*}$ and $\zeta_{n} \rightarrow \zeta^{*}$. We can verify that $\zeta^{*} \in \Upsilon_{2} \varrho^{*}$. Indeed, for each $n \geq 1$ and $\zeta_{n} \in \Upsilon_{2} \varrho_{n}$, select $\vartheta_{n} \in(\mathcal{S E L})_{\Psi, \varrho_{n}}$ such that

$$
\begin{aligned}
\zeta_{n}(t)= & \int_{0}^{t} \frac{(t-\tau)^{\nu-1}}{\Gamma(\nu)} \vartheta_{n}(\tau) \mathrm{d} \tau-\frac{t}{3} \int_{0}^{1} \frac{(1-\tau)^{\nu-1}}{\Gamma(\nu)} \vartheta_{n}(\tau) \mathrm{d} \tau \\
& -\frac{t}{3} \int_{0}^{1} \frac{(1-\tau)^{\nu-2}}{\Gamma(\nu-1)} \vartheta_{n}(\tau) \mathrm{d} \tau+\frac{\left(t-t^{2}\right) \Gamma(3-\theta)}{2+4 \Gamma(3-\theta)} \int_{0}^{1} \frac{(1-\tau)^{\nu-3}}{\Gamma(\nu-2)} \vartheta_{n}(\tau) \mathrm{d} \tau \\
& +\frac{\left(t-t^{2}\right) \Gamma(3-\theta)}{2+4 \Gamma(3-\theta)} \int_{0}^{1} \frac{(1-\tau)^{\nu-\theta-1}}{\Gamma(\nu-\theta)} \vartheta_{n}(\tau) \mathrm{d} \tau
\end{aligned}
$$

for any $t \in[0,1]$. In this case, we have to show that there is $v^{*} \in(\mathcal{S E L})_{\Psi, e^{*}}$ such that

$$
\begin{aligned}
\zeta^{*}(t)= & \int_{0}^{t} \frac{(t-\tau)^{\nu-1}}{\Gamma(\nu)} \vartheta^{*}(\tau) \mathrm{d} \tau-\frac{t}{3} \int_{0}^{1} \frac{(1-\tau)^{\nu-1}}{\Gamma(\nu)} \vartheta^{*}(\tau) \mathrm{d} \tau \\
& -\frac{t}{3} \int_{0}^{1} \frac{(1-\tau)^{\nu-2}}{\Gamma(\nu-1)} \vartheta^{*}(\tau) \mathrm{d} \tau+\frac{\left(t-t^{2}\right) \Gamma(3-\theta)}{2+4 \Gamma(3-\theta)} \int_{0}^{1} \frac{(1-\tau)^{\nu-3}}{\Gamma(\nu-2)} \vartheta^{*}(\tau) \mathrm{d} \tau
\end{aligned}
$$




$$
+\frac{\left(t-t^{2}\right) \Gamma(3-\theta)}{2+4 \Gamma(3-\theta)} \int_{0}^{1} \frac{(1-\tau)^{\nu-\theta-1}}{\Gamma(\nu-\theta)} \vartheta^{*}(\tau) \mathrm{d} \tau
$$

for all $t \in[0,1]$. Define the continuous linear operator $\Xi: \mathcal{L}_{\mathbb{R}}^{1}([0,1]) \rightarrow \mathcal{X}=C_{\mathbb{R}}([0,1])$ by

$$
\begin{aligned}
\Xi(\vartheta)(t)=\varrho(t)= & \int_{0}^{t} \frac{(t-\tau)^{\nu-1}}{\Gamma(\nu)} \vartheta(\tau) \mathrm{d} \tau-\frac{t}{3} \int_{0}^{1} \frac{(1-\tau)^{\nu-1}}{\Gamma(\nu)} \vartheta(\tau) \mathrm{d} \tau \\
& -\frac{t}{3} \int_{0}^{1} \frac{(1-\tau)^{\nu-2}}{\Gamma(v-1)} \vartheta(\tau) \mathrm{d} \tau+\frac{\left(t-t^{2}\right) \Gamma(3-\theta)}{2+4 \Gamma(3-\theta)} \int_{0}^{1} \frac{(1-\tau)^{\nu-3}}{\Gamma(\nu-2)} \vartheta(\tau) \mathrm{d} \tau \\
& +\frac{\left(t-t^{2}\right) \Gamma(3-\theta)}{2+4 \Gamma(3-\theta)} \int_{0}^{1} \frac{(1-\tau)^{\nu-\theta-1}}{\Gamma(\nu-\theta)} \vartheta(\tau) \mathrm{d} \tau
\end{aligned}
$$

for each $t \in[0,1]$. Then we have

$$
\begin{aligned}
\left\|\zeta_{n}(t)-\zeta^{*}(t)\right\|= & \| \int_{0}^{t} \frac{(t-\tau)^{\nu-1}}{\Gamma(v)}\left(\vartheta_{n}(\tau)-\vartheta^{*}(\tau)\right) \mathrm{d} \tau \\
& -\frac{t}{3} \int_{0}^{1} \frac{(1-\tau)^{\nu-1}}{\Gamma(v)}\left(\vartheta_{n}(\tau)-\vartheta^{*}(\tau)\right) \mathrm{d} \tau \\
& -\frac{t}{3} \int_{0}^{1} \frac{(1-\tau)^{\nu-2}}{\Gamma(\nu-1)}\left(\vartheta_{n}(\tau)-\vartheta^{*}(\tau)\right) \mathrm{d} \tau \\
& +\frac{\left(t-t^{2}\right) \Gamma(3-\theta)}{2+4 \Gamma(3-\theta)} \int_{0}^{1} \frac{(1-\tau)^{\nu-3}}{\Gamma(v-2)}\left(\vartheta_{n}(\tau)-\vartheta^{*}(\tau)\right) \mathrm{d} \tau \\
& +\frac{\left(t-t^{2}\right) \Gamma(3-\theta)}{2+4 \Gamma(3-\theta)} \int_{0}^{1} \frac{(1-\tau)^{\nu-\theta-1}}{\Gamma(\nu-\theta)}\left(\vartheta_{n}(\tau)-\vartheta^{*}(\tau)\right) \mathrm{d} \tau \|
\end{aligned}
$$

Letting $n \rightarrow \infty$, the above estimate yields $\left\|\zeta_{n}(t)-\zeta^{*}(t)\right\| \rightarrow 0$. Hence with due attention to Theorem 2 , one can deduce that $\Xi \circ(\mathcal{S E} \mathcal{L})_{\Psi}$ is an operator having a closed graph property. As $\zeta_{n} \in \Xi\left((\mathcal{S E L})_{\Psi, \varrho_{n}}\right)$ and $\varrho_{n} \rightarrow \varrho^{*}$, so there exists $\vartheta^{*} \in(\mathcal{S E} \mathcal{L})_{\Psi, \varrho^{*}}$ such that

$$
\begin{aligned}
\zeta^{*}(t)= & \int_{0}^{t} \frac{(t-\tau)^{\nu-1}}{\Gamma(\nu)} \vartheta^{*}(\tau) \mathrm{d} \tau-\frac{t}{3} \int_{0}^{1} \frac{(1-\tau)^{\nu-1}}{\Gamma(\nu)} \vartheta^{*}(\tau) \mathrm{d} \tau \\
& -\frac{t}{3} \int_{0}^{1} \frac{(1-\tau)^{\nu-2}}{\Gamma(v-1)} \vartheta^{*}(\tau) \mathrm{d} \tau+\frac{\left(t-t^{2}\right) \Gamma(3-\theta)}{2+4 \Gamma(3-\theta)} \int_{0}^{1} \frac{(1-\tau)^{\nu-3}}{\Gamma(v-2)} \vartheta^{*}(\tau) \mathrm{d} \tau \\
& +\frac{\left(t-t^{2}\right) \Gamma(3-\theta)}{2+4 \Gamma(3-\theta)} \int_{0}^{1} \frac{(1-\tau)^{\nu-\theta-1}}{\Gamma(v-\theta)} \vartheta^{*}(\tau) \mathrm{d} \tau
\end{aligned}
$$

for all $t \in[0,1]$. Hence, $\zeta^{*} \in \Upsilon_{2} \varrho^{*}$ and so $\Upsilon_{2}$ has a closed graph. Therefore, the upper semicontinuity of the operator $\Upsilon_{2}$ is fulfilled. By utilizing the assumption of theorem, we know that $\Upsilon_{2}$ has compact values. Consequently, $\Upsilon_{2}$ is an upper semi-continuous and compact operator. Now, under assumption $\left(\mathcal{A}^{\prime} 3\right)$, we have

$$
\begin{aligned}
\mathcal{K}_{0}^{*}= & \left\|\Upsilon_{2}(\mathcal{X})\right\| \\
= & \sup _{t \in[0,1]}\left\{\left|\Upsilon_{2} \varrho\right|: \varrho \in \mathcal{X}\right\} \\
= & {\left[\frac{4+v}{3 \Gamma(v+1)}+\frac{\Gamma(3-\theta)}{(2+4 \Gamma(3-\theta)) \Gamma(v-1)}+\frac{\Gamma(3-\theta)}{(2+4 \Gamma(3-\theta)) \Gamma(v-\theta+1)}\right] } \\
& \times\|\sigma\|_{\mathcal{L}^{1}}=\Delta^{*}\|\sigma\|_{\mathcal{L}^{1}} .
\end{aligned}
$$


By putting $l^{*}=s^{*}$, we obtain $l^{*} \mathcal{K}_{0}^{*}<\frac{1}{2}$. Hence, all the assumptions of Theorem 3 hold for $\Upsilon_{1}$. Here, we claim that only one of conditions $\left(b^{\prime} 1\right)$ or $\left(b^{\prime} 2\right)$ is valid. By applying Theorem 3 and assumption $\left(\mathcal{A}^{\prime} 4\right)$, consider an arbitrary element $\varrho$ of $\mathcal{Y}^{*}$ with $\|\varrho\|=\tilde{\varepsilon}$. Then $\mu \varrho(t) \in$ $\Upsilon_{1} \varrho(t) \Upsilon_{2} \varrho(t)$ for each $\mu>1$. Select the related function $\vartheta \in(\mathcal{S E L})_{\Psi, \varrho}$. Then, for each $\mu>1$, we obtain

$$
\begin{aligned}
\varrho(t)= & \frac{1}{\mu} w(t, \varrho(t))\left[\int_{0}^{t} \frac{(t-\tau)^{\nu-1}}{\Gamma(\nu)} \vartheta(\tau) \mathrm{d} \tau-\frac{t}{3} \int_{0}^{1} \frac{(1-\tau)^{\nu-1}}{\Gamma(\nu)} \vartheta(\tau) \mathrm{d} \tau\right. \\
& -\frac{t}{3} \int_{0}^{1} \frac{(1-\tau)^{\nu-2}}{\Gamma(\nu-1)} \vartheta(\tau) \mathrm{d} \tau+\frac{\left(t-t^{2}\right) \Gamma(3-\theta)}{2+4 \Gamma(3-\theta)} \int_{0}^{1} \frac{(1-\tau)^{\nu-3}}{\Gamma(\nu-2)} \vartheta(\tau) \mathrm{d} \tau \\
& \left.+\frac{\left(t-t^{2}\right) \Gamma(3-\theta)}{2+4 \Gamma(3-\theta)} \int_{0}^{1} \frac{(1-\tau)^{\nu-\theta-1}}{\Gamma(\nu-\theta)} \vartheta(\tau) \mathrm{d} \tau\right]
\end{aligned}
$$

for all $t \in[0,1]$. Thus, we have

$$
\begin{aligned}
|\varrho(t)|= & \frac{1}{\mu}|w(t, \varrho(t))|\left[\int_{0}^{t} \frac{(t-\tau)^{\nu-1}}{\Gamma(v)}|\vartheta(\tau)| \mathrm{d} \tau-\frac{t}{3} \int_{0}^{1} \frac{(1-\tau)^{\nu-1}}{\Gamma(v)}|\vartheta(\tau)| \mathrm{d} \tau\right. \\
& -\frac{t}{3} \int_{0}^{1} \frac{(1-\tau)^{\nu-2}}{\Gamma(v-1)}|\vartheta(\tau)| \mathrm{d} \tau+\frac{\left|t-t^{2}\right| \Gamma(3-\theta)}{2+4 \Gamma(3-\theta)} \int_{0}^{1} \frac{(1-\tau)^{\nu-3}}{\Gamma(v-2)}|\vartheta(\tau)| \mathrm{d} \tau \\
& \left.+\frac{\left|t-t^{2}\right| \Gamma(3-\theta)}{2+4 \Gamma(3-\theta)} \int_{0}^{1} \frac{(1-\tau)^{\nu-\theta-1}}{\Gamma(v-\theta)}|\vartheta(\tau)| \mathrm{d} \tau\right] \\
\leq & {[|w(t, \varrho(t))-w(t, 0)|+|w(t, 0)|] } \\
& \times\left[\int_{0}^{t} \frac{(t-\tau)^{\nu-1}}{\Gamma(v)}|\vartheta(\tau)| \mathrm{d} \tau-\frac{t}{3} \int_{0}^{1} \frac{(1-\tau)^{\nu-1}}{\Gamma(v)}|\vartheta(\tau)| \mathrm{d} \tau\right. \\
& -\frac{t}{3} \int_{0}^{1} \frac{(1-\tau)^{\nu-2}}{\Gamma(\nu-1)}|\vartheta(\tau)| \mathrm{d} \tau+\frac{\left|t-t^{2}\right| \Gamma(3-\theta)}{2+4 \Gamma(3-\theta)} \int_{0}^{1} \frac{(1-\tau)^{\nu-3}}{\Gamma(v-2)}|\vartheta(\tau)| \mathrm{d} \tau \\
& \left.+\frac{\left|t-t^{2}\right| \Gamma(3-\theta)}{2+4 \Gamma(3-\theta)} \int_{0}^{1} \frac{(1-\tau)^{\nu-\theta-1}}{\Gamma(v-\theta)}|\vartheta(\tau)| \mathrm{d} \tau\right] \\
\leq & {\left[s^{*}\|\varrho\|+W^{*}\right]\left[\int_{0}^{t} \frac{(t-\tau)^{\nu-1}}{\Gamma(v)} \sigma(\tau) \mathrm{d} \tau-\frac{t}{3} \int_{0}^{1} \frac{(1-\tau)^{\nu-1}}{\Gamma(\nu)} \sigma(\tau) \mathrm{d} \tau\right.} \\
& -\frac{t}{3} \int_{0}^{1} \frac{(1-\tau)^{\nu-2}}{\Gamma(v-1)} \sigma(\tau) \mathrm{d} \tau+\frac{\left|t-t^{2}\right| \Gamma(3-\theta)}{2+4 \Gamma(3-\theta)} \int_{0}^{1} \frac{(1-\tau)^{\nu-3}}{\Gamma(v-2)} \sigma(\tau) \mathrm{d} \tau \\
& \left.+\frac{\left|t-t^{2}\right| \Gamma(3-\theta)}{2+4 \Gamma(3-\theta)} \int_{0}^{1} \frac{(1-\tau)^{\nu-\theta-1}}{\Gamma(v-\theta)} \sigma(\tau) \mathrm{d} \tau\right] \\
\leq & {\left[s^{*} \tilde{\varepsilon}+W^{*}\right] \Delta^{*}\|\sigma\| \mathcal{L}^{1} }
\end{aligned}
$$

for any $t \in[0,1]$. By simple computations, we get $\tilde{\varepsilon} \leq \frac{W^{*} \Delta^{*}\|\sigma\|}{1-s^{*} \Delta^{*}\|\sigma\|_{\mathcal{L}^{1}}}$. According to condition (11), we find that condition $\left(b^{\prime} 1\right)$ of Theorem 3 is not possible and condition $\left(b^{\prime} 2\right)$ is valid. Therefore, the operator inclusion $\varrho \in \Upsilon_{1} \varrho \Upsilon_{2} \varrho$ has a solution, and so the hybrid inclusion BVP (3)-(4) has a solution. This ends the proof.

To demonstrate the consistency and the applicability of the obtained results, two illustrative numerical examples are provided herein. 
Example 1 Corresponding to the proposed hybrid BVP (1)-(2), we define the hybrid differential equation of fractional order

$$
{ }^{c} \mathcal{D}^{2.74}\left(\frac{\varrho(t)}{\frac{0.3 t|\varrho(t)|^{4}}{7+|\varrho(t)|^{4}}+0.378}\right)=\frac{t \sin (\varrho(t)) \cos ^{2}(\pi(t))}{3040} \quad(t \in[0,1])
$$

with hybrid boundary value conditions

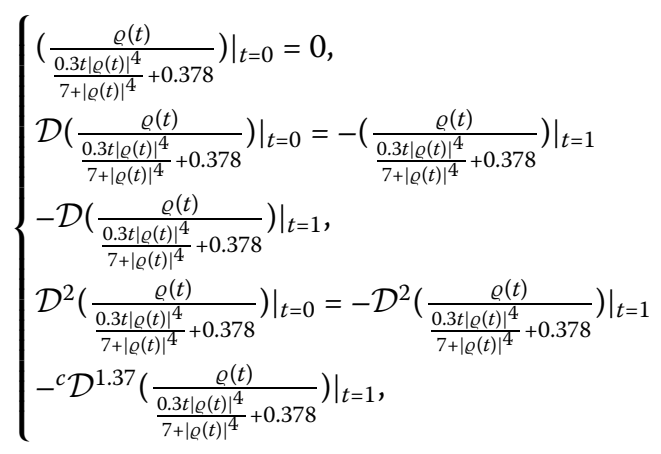

where $v=2.74$ and $\theta=1.37$. Consider the nonzero continuous real-valued map $w$ on $[0,1] \times \mathbb{R}$ defined by $w(t, \varrho(t))=\frac{0.3 t|\varrho(t)|^{4}}{7+|\varrho(t)|^{4}}+0.378$ with $W^{*}=\sup _{t \in[0,1]}|w(t, 0)|=0.378$. Define the continuous map $\Phi:[0,1] \times \mathbb{R} \rightarrow \mathbb{R}^{+}$by $\Phi(t, \varrho(t))=\frac{t \sin (\varrho(t)) \cos ^{2}(\pi(t))}{3040}$. As $s(t)=$ $0.3 t$, so we get $s^{*}=\sup _{t \in[0,1]}|\kappa(t)|=0.3$. Put $h(t)=\frac{t}{3040}$. Hence $H^{*}=\sup _{t \in[0,1]}|h(t)|=$ $\frac{1}{3040}$ and $\xi(\|\varrho\|)=1$. Thus, we obtain $\Delta^{*} \simeq 0.8227$. Choose $\varepsilon>0.0000995$. In this case, $s^{*} \Delta^{*} H^{*} \xi(\|\rho\|) \simeq 0.0000078<1$. Hence all the hypotheses of Theorem 5 are valid, and so the hybrid differential equation (12)-(13) has a solution.

Example 2 Corresponding to the proposed hybrid BVP (3)-(4), we consider the hybrid inclusion problem of fractional order

$$
\begin{aligned}
& { }^{c} \mathcal{D}^{2.83}\left(\frac{\varrho(t)}{\frac{t}{200} \sin \varrho(t)+89}\right) \in\left[\frac{|\varrho(t)|}{6(1+|\varrho(t)|)}+\frac{1}{2}, \frac{|\cos \varrho(t)|}{7(1+|\cos \varrho(t)|)}+\frac{6}{7}\right] \\
& (t \in[0,1])
\end{aligned}
$$

with hybrid boundary value conditions

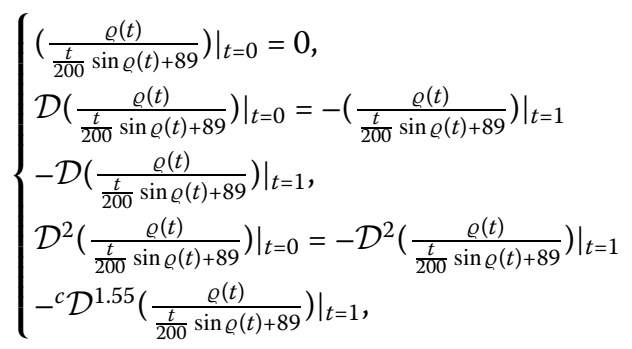

where $v=2.83$ and $\theta=1.55$. Define the continuous map $w:[0,1] \times \mathbb{R} \rightarrow \mathbb{R} \backslash\{0\}$ by $w(t, \varrho(t))=\frac{t}{200} \sin \varrho(t)+89$ with $W^{*}=\sup _{t \in[0,1]}|w(t, 0)|=89$. Further, we define the setvalued map $\Psi:[0,1] \times \mathbb{R} \rightarrow \mathcal{P}(\mathbb{R})$ by $\Psi(t, \varrho(t))=\left[\frac{|\varrho(t)|}{6(1+|\varrho(t)|)}+\frac{1}{2}, \frac{|\cos \varrho(t)|}{7(1+|\cos \varrho(t)|)}+\frac{6}{7}\right]$. If $s(t)=\frac{t}{200}$, then $s^{*}=\sup _{t \in[0,1]}|s(t)|=\frac{1}{200}$. As $|\zeta| \leq \max \left[\frac{|\varrho(t)|}{6(1+|\varrho(t)|)}+\frac{1}{2}, \frac{|\cos \varrho(t)|^{3}}{7\left(1+|\cos \varrho(t)|^{3}\right)}+\frac{6}{7}\right] \leq 1$ for all $\varrho \in \mathbb{R}$ 
and $\zeta \in \Psi(t, \varrho(t))$, we get $\|\Psi(t, \varrho(t))\|=\sup \{|\vartheta|: \vartheta \in \Psi(t, \varrho(t))\} \leq 1$. Setting $\sigma(t)=1$ for all $t \in[0,1]$, we obtain $\|\sigma\|_{\mathcal{L}^{1}}=1$ and $\Delta^{*} \simeq 0.76$. We can find $\tilde{\varepsilon}>0$ with $\tilde{\varepsilon}>67.898$. Note

that $s^{*} \Delta^{*}\|\sigma\|_{\mathcal{L}^{1}} \simeq 0.0038<\frac{1}{2}$. In view of Theorem 7 , we conclude that the hybrid inclusion BVP of fractional order (14)-(15) has a solution.

\title{
4 Conclusion
}

Nowadays, it is a vital goal that we could model most phenomena in the real world. For example, modeling of chemical reactions using some modern software to reduce the use of materials in chemical laboratories. This will contribute to environmental protection. Thus, we should endeavor to increase our creativity to study the complicated modeling of differential equations and inclusions. In the present research work, we design a novel fractional hybrid differential equation and its related inclusion version with hybrid conditions. To reach the desired findings, some analytical techniques are adopted from the concepts of nonlinear analysis. Finally, to demonstrate the consistency and applicability of the obtained results, two illustrative numerical examples are provided. We believe that our hybrid problems involve some particular cases, which can extend to more general hybrid problems. The fractional hybrid modeling is of great significance in different engineering fields, and it can be a unique idea for the future combined research between various applied sciences. So we leave the new abstract idea for interested researchers as future projects.

\author{
Acknowledgements \\ The first and third authors were supported by Azarbaijan Shahid Madani University. The authors express their gratitude to \\ dear unknown referees for their helpful suggestions which improved the final version of this paper. \\ Funding \\ Not applicable. \\ Availability of data and materials \\ Data sharing not applicable to this article as no datasets were generated or analyzed during the current study. \\ Ethics approval and consent to participate \\ Not applicable. \\ Competing interests \\ The authors declare that they have no competing interests. \\ Consent for publication \\ Not applicable. \\ Authors' contributions \\ The authors declare that the study was realized in collaboration with equal responsibility. All authors read and approved \\ the final manuscript. \\ Author details \\ 'Department of Mathematics, Azarbaijan Shahid Madani University, Tabriz, Iran. ${ }^{2}$ Department of Mathematics, Shabestar \\ Branch, Islamic Azad University, Shabestar, Iran. ${ }^{3}$ Department of Medical Research, China Medical University Hospital, \\ China Medical University, Taichung, Taiwan.
}

\section{Publisher's Note}

Springer Nature remains neutral with regard to jurisdictional claims in published maps and institutional affiliations.

Received: 5 April 2020 Accepted: 10 June 2020 Published online: 19 June 2020

References

1. Alizadeh, S., Baleanu, D., Rezapour, S.: Analyzing transient response of the parallel RCL circuit by using the Caputo-Fabrizio fractional derivative. Adv. Differ. Equ. 2020, 55 (2020). https://doi.org/10.1186/s13662-020-2527-0

2. Baleanu, D., Jajarmi, A., Mohammadi, H., Rezapour, S.: Analysis of the human liver model with Caputo-Fabrizio fractional derivative. Chaos Solitons Fractals 134, 7 (2020) 
3. Baleanu, D., Mohammadi, H., Rezapour, S.: Mathematical theoretical study of a particular system of Caputo-Fabrizio fractional differential equations for the rubella disease model. Adv. Differ. Equ. 2020, 184 (2020). https://doi.org/10.1186/s13662-020-02614-z

4. Baleanu, D., Mohammadi, H., Rezapour, S.: Analysis of the model of HIV-1 infection of $c d 4^{+}$T-cell with a new approach of fractional derivative. Adv. Differ. Equ. 2020, 71 (2020)

5. Kumar, D., Singh, J., Baleanu, D.: On the analysis of vibration equation involving a fractional derivative with Mittag-Leffler law. Math. Methods Appl. Sci. 43(1), 443-457 (2019). https://doi.org/10.1002/mma.5903

6. Bhatter, S., Mathur, A., Kumar, D., Nisar, K.S., Singh, J.: Fractional modified Kawahara equation with Mittag-Leffler law. Chaos Solitons Fractals 131, 109508 (2020). https://doi.org/10.1016/j.chaos.2019.109508

7. Kumar, D., Singh, J., Tanwar, K., Baleanu, D.: A new fractional exothermic reactions model having constant heat source in porous media with power, exponential and Mittag-Leffler laws. Int. J. Heat Mass Transf. 138, 1222-1227 (2019). https://doi.org/10.1016/j.ijheatmasstransfer.2019.04.094

8. Goswami, A., Singh, J., Kumar, D., Sunshila: An efficient analytical approach for fractional equal width equations describing hydro-magnetic waves in cold plasma. Phys. A, Stat. Mech. Appl. 524, 563-575 (2019) https://doi.org/10.1016/j.physa.2019.04.058

9. Singh, J., Kumar, D., Baleanu, D.: A new analysis of fractional fish farm model associated with Mittag-Leffler type kernel. Int. J. Biomath. 13(2), 2050010 (2020). https://doi.org/10.1142/S1793524520500102

10. Ma, C.Y., Shiri, B., Wu, G.C., Baleanu, D.: New signal smoothing equations with short memory and variable order. Optik 218, 164507 (2020). https://doi.org/10.1016/j.ijleo.2020.164507

11. Alijani, Z., Baleanu, D., Shiri, B., Wu, G.: Spline collocation methods for systems of fuzzy fractional differential equations. Chaos Solitons Fractals 131, 109510 (2020)

12. Dadkhah, E., Shiri, B., Ghaffarzadeh, H., Baleanu, D.: Visco-elastic dampers in structural buildings and numerical solution with spline collocation methods. J. Appl. Math. Comput. 63, 29-57 (2020)

13. Shiri, B., Baleanu, D.: System of fractional differential algebraic equations with applications. Chaos Solitons Fractals 120, 203-212 (2019)

14. Dadkhah, E., Ghaffarzadeh, H., Shiri, B., Katebi, J.: Spline collocation methods for seismic analysis of multiple degree of freedom systems with visco-elastic dampers using fractional models. J. Vib. Control (2020)

15. Baleanu, D., Shiri, B.: Collocation methods for fractional differential equations involving non-singular kernel. Chaos Solitons Fractals 116, 136-145 (2018)

16. Baleanu, D., Ghafarnezhad, K., Rezapour, S.: On a three steps crisis integro-differential equation. Adv. Differ. Equ. 2019, 153 (2019)

17. Baleanu, D., Rezapour, S., Mohammadi, H.: Some existence results on nonlinear fractional differential equations Philos. Trans. R. Soc. A, Math. Phys. Eng. Sci. 371, 20120144 (2013). https://doi.org/10.1098/rsta.2012.0144

18. Aydogan, M., Baleanu, D., Mousalou, A., Rezapour, S.: On high order fractional integro-differential equations including the Caputo-Fabrizio derivative. Bound. Value Probl. 2018, 90 (2018). https://doi.org/10.1186/s13661-018-1008-9

19. Aydogan, M., Baleanu, D., Mousalou, A., Rezapour, S.: On approximate solutions for two higher-order Caputo-Fabrizio fractional integro-differential equations. Adv. Differ. Equ. 2017, 221 (2017). https://doi.org/10.1186/s13662-017-1258-3

20. Baleanu, D., Rezapour, S., Saberpour, Z:: On fractional integro-differential inclusions via the extended fractional Caputo-Fabrizio derivation. Bound. Value Probl. 2019, 79 (2019)

21. Delbosco, D.: Fractional calculus and function spaces. J. Fract. Calc. 6, 45-53 (1994)

22. Zhang, S.: The existence of a positive solution for a nonlinear fractional differential equation. J. Math. Anal. Appl. 252(2), 804-812 (2000). https://doi.org/10.1006/jmaa.2000.7123

23. Bai, Z., Lü, H.: Positive solutions for boundary value problem of nonlinear fractional differential equation. J. Math. Anal. Appl. 311(2), 495-505 (2005). https://doi.org/10.1016/j.jmaa.2005.02.052

24. Bai, Z., Lü, H.: Positive solutions for boundary value problem of nonlinear fractional differential equation. J. Math. Anal. Appl. 311(2), 495-505 (2005). https://doi.org/10.1016/j.jmaa.2005.02.052

25. Kochubei, A.N.: Distributed order calculus and equations of ultraslow diffusion. J. Math. Anal. Appl. 340(1), 252-281 (2008). https://doi.org/10.1016/j.jmaa.2007.08.024

26. Leggett, R.W., Williams, L.R.: Multiple positive fixed points of nonlinear operators on ordered Banach spaces. Indiana Univ. Math. J. 28(4), 673-688 (1979)

27. Agarwal, R.P., O'Regan, D., Staněk, S.: The existence of solutions for a nonlinear mixed problem of singular fractional differential equations. Math. Nachr. 285(1), 27-41 (2012). https://doi.org/10.1002/mana.201000043

28. Agarwal, R.P., O'Regan, D., Staněk, S.: Positive solutions for Dirichlet problems of singular nonlinear fractional differential equations. J. Math. Anal. Appl. 371(1), 57-68 (2010). https://doi.org/10.1016/j.jmaa.2010.04.034

29. Jiang, M., Zhong, S.: Existence of solutions for nonlinear fractional q-difference equations with Riemann-Liouville type q-derivatives. J. Appl. Math. Comput. 47(1-2), 429-459 (2015). https://doi.org/10.1007/s12190-014-0784-3

30. Zhang, X., Zhong, Q.: Multiple positive solutions for nonlocal boundary value problems of singular fractional differential equations. Bound. Value Probl. 2016, 65 (2016). https://doi.org/10.1186/s13661-016-0572-0

31. Zhou, H., Alzabut, J., Yang, L.: On fractional Langevin differential equations with anti-periodic boundary conditions. Eur. Phys. J. Spec. Top. 226, 3577-3590 (2017). https://doi.org/10.1140/epjst/e2018-00082-0

32. Xu, X., Jiang, D., Yuan, C.: Multiple positive solutions for the boundary value problem of a nonlinear fractional differential equation. Nonlinear Anal. 71,4676-4688 (2009). https://doi.org/10.1016/j.na.2009.03.030

33. Ahmad, B., Nieto, J.J.: Riemann-Liouville fractional integro-differential equations with fractional nonlocal integral boundary conditions. Bound. Value Probl. 2011, 36 (2011). https://doi.org/10.1186/1687-2770-2011-36

34. Su, X., Zhang, S.: Solutions to boundary value problems for nonlinear differential equations of fractional order Electron. J. Differ. Equ. 2009(26), 1 (2009)

35. Ragusa, M.A.: Cauchy-Dirichlet problem associated to divergence form parabolic equations. Commun. Contemp. Math. 6(3), 377-393 (2004). https://doi.org/10.1142/S0219199704001392

36. Chidouh, A., Torres, D.: Existence of positive solutions to a discrete fractional boundary value problem and corresponding Lyapunov-type inequalities. Opusc. Math. 38(1)، 31-40 (2018).

https://doi.org/10.7494/OpMath.2018.38.1.31 
37. Denton, Z., Ramírez, J.D.: Existence of minimal and maximal solutions to RL fractional integro-differential initial value problems. Opusc. Math. 37(5), 705-724 (2017). https://doi.org/10.7494/OpMath.2017.37.5.705

38. Liu, Y: A new method for converting boundary value problems for impulsive fractional differential equations to integral equations and its applications. Adv. Nonlinear Anal. 8(1), 386-454 (2019). https://doi.org/10.1515/anona-2016-0064

39. Wang, Y., Liu, L.: Necessary and sufficient condition for the existence of positive solution to singular fractional differential equations. Adv. Differ. Equ. 2015, 207 (2015)

40. Wang, Y: Positive solutions for a class of two-term fractional differential equations with multipoint boundary value conditions. Adv. Differ. Equ. 2019, 304 (2019). https://doi.org/10.1186/s13662-019-2250-x

41. Wang, Y.: Necessary conditions for the existence of positive solutions to fractional boundary value problems at resonance. Appl. Math. Lett. 97, 34-40 (2019). https://doi.org/10.1016/j.aml.2019.05.007

42. Bungardi, S., Cardinali, T., Rubbioni, P.: Nonlocal semi-linear integro-differential inclusions via vectorial measures of non-compactness. Appl. Anal. 96(15), 2526-2544 (2015)

43. Ndaírou, F., Area, I., Nieto, J.J., Torres, D.F.M.: Mathematical modeling of COVID-19 transmission dynamics with a case study of Wuhan. Chaos Solitons Fractals 135, 109846 (2020)

44. Kucche, K.D., Nieto, J.J., Venktesh, V.: Theory of nonlinear implicit fractional differential equations. Differ. Equ. Dyn. Syst. 28(1), 1-17 (2020)

45. Ahmad, B., Alruwaily, Y., Alsaedi, A., Nieto, J.J.: Fractional integro-differential equations with dual anti-periodic boundary conditions. Differ. Integral Equ. 33(3-4), 181-206 (2020)

46. Nisar, K.S., Suthar, D.L., Agarwal, R., Purohit, S.D.: Fractional calculus operators with Appell function kernels applied to Srivastava polynomials and extended Mittag-Leffler function. Adv. Differ. Equ. 2020, 148 (2020)

47. Agarwal, R., Golev, A., Hristova, S., O'Regan, D., Stefanova, K.: Iterative techniques with computer realization for the initial value problem for Caputo fractional differential equations. J. Appl. Math. Comput. 58(1-2), 433-467 (2018)

48. Hristova, S., Agarwal, R., O'Regan, D.: Explicit solutions of initial value problems for systems of linear Riemann-Liouville fractional differential equations with constant delay. Adv. Differ. Equ. 2020, 180 (2020)

49. Wang, X., Li, X., Huang, N., O'Regan, D.: Asymptotical consensus of fractional-order multi-agent systems with current and delay states. Appl. Math. Mech. 40(11), 1677-1694 (2019)

50. Song, J., Xia, Y., Bai, Y., Cai, Y., O'Regan, D.: A non-autonomous Leslie-Gower model with Holling type IV functiona response and harvesting complexity. Adv. Differ. Equ. 2019, 299 (2019)

51. Agarwal, P., Chand, M., Baleanu, D., O'Regan, D., Jain, S.: On the solutions of certain fractional kinetic equations involving k-Mittag-Leffler function. Adv. Differ. Equ. 2018, 249 (2018)

52. Dhage, B.C., Lakshmikantham, V:: Basic results on hybrid differential equation. Nonlinear Anal. Hybrid Syst. 4, 414-424 (2010)

53. Zhao, Y., Sun, S., Han, Z., Li, Q.: Theory of fractional hybrid differential equations. Comput. Math. Appl. 62(3), 1312-1324 (2011). https://doi.org/10.1016/j.camwa.2011.03.041

54. Hilal, K., Kajouni, A.: Boundary value problems for hybrid differential equations with fractional order. Adv. Differ. Equ. 2015, $183(2015)$

55. Baleanu, D., Hedayati, V., Rezapour, S., Al Qurashi, M.M.: On two fractional differential inclusions. SpringerPlus 5(1), 882 (2016). https://doi.org/10.1186/s40064-016-2564-Z

56. Derbazi, C., Hammouche, H., Benchohra, M., Zhou, Y.: Fractional hybrid differential equations with three-point boundary hybrid conditions. Adv. Differ. Equ. 2019, 125 (2019)

57. Baleanu, D., Etemad, S., Pourrazi, S., Rezapour, S.: On the new fractional hybrid boundary value problems with three-point integral hybrid conditions. Adv. Differ. Equ. 2019, 473 (2019)

58. Baleanu, D., Etemad, S., Rezapour, S.: A hybrid Caputo fractional modeling for thermostat with hybrid boundary value conditions. Bound. Value Probl. 2020, 64 (2020)

59. Podlubny, l.: Fractional Differential Equations. Academic Press, San Diego (1999)

60. Samko, S.G., Kilbas, A.A., Marichev, O.I.: Fractional Integrals and Derivatives: Theory and Applications. Gordon \& Breach, Philadelphia (1993)

61. Deimling, K:: Multi-Valued Differential Equations. de Gruyter, Berlin (1992)

62. Aubin, J., Ceuina, A.: Differential Inclusions: Set-Valued Maps and Viability Theory. Springer, Berlin (1984). https://doi.org/10.1007/978-3-642-69512-4

63. Dhage, B.C.: Nonlinear functional boundary value problems involving Carathédory. Kyungpook Math. J. 46, 427-441 (2006)

64. Lasota, A., Opial, Z:: An application of the Kakutani-Ky Fan theorem in the theory of ordinary differential equations. Bull. Acad. Pol. Sci. Set. Sci. Math. Astronom. Phy. 13, 781-786 (1965)

65. Dhage, B.C.: Existence results for neutral functional differential inclusions in Banach algebras. Nonlinear Anal. 64, 1290-1306 (2006) 